Portland State University

PDXScholar

Spring 7-5-2013

\title{
A Parabolic Equation Analysis of the Underwater Noise Radiated by Impact Pile Driving
}

Nathan Laws

Portland State University

Follow this and additional works at: https://pdxscholar.library.pdx.edu/open_access_etds

Part of the Other Electrical and Computer Engineering Commons, and the Other Mathematics Commons

Let us know how access to this document benefits you.

\section{Recommended Citation}

Laws, Nathan, "A Parabolic Equation Analysis of the Underwater Noise Radiated by Impact Pile Driving" (2013). Dissertations and Theses. Paper 1083.

https://doi.org/10.15760/etd.1083

This Thesis is brought to you for free and open access. It has been accepted for inclusion in Dissertations and Theses by an authorized administrator of PDXScholar. Please contact us if we can make this document more accessible: pdxscholar@pdx.edu. 
A Parabolic Equation Analysis of the Underwater Noise Radiated by Impact Pile Driving

by

Nathan Laws

A thesis submitted in partial fulfillment of the requirements for the degree of

\author{
Master of Science \\ in \\ Electrical and Computer Engineering
}

Thesis Committee:

Lisa M. Zurk, Chair

Martin Siderius

Daniel Rouseff

Portland State University

2013 
(c)2013 Nathan Laws 


\begin{abstract}
Impact pile driving can produce extremely high underwater sound levels, which are of increasing environmental concern due to their deleterious effects on marine wildlife. Prediction of underwater sound levels is important to the assessment and mitigation of the environmental impacts caused by pile driving. Current prediction methods are limited and do not account for the dynamic pile driving source, inhomogeneities in bathymetry and sediment, or physics-based sound wave propagation.

In this thesis, a computational model is presented that analyzes and predicts the underwater noise radiated by pile driving and is suitable for shallow, inhomogeneous environments and long propagation ranges. The computational model uses dynamic source models from recent developments in the technical literature. Pile source models are coupled to a broadband application of the range-dependent acoustic model (RAM-PE), a standard parabolic equation (PE) propagation code capable of modeling wave propagation through complex, range dependent environments. Simulation results are shown to be in good agreement with several observations of pile driving operations in the Columbia River between Portland, Oregon and Vancouver, Washington. The model is further applied to extend sound level predictions over the entire river and study the effects of sediment and bathymetry on the underwater sound levels present in the environment.
\end{abstract}




\section{Acknowledgments}

I would like to thank my advisor Dr. Lisa Zurk for her guidance and support during this research. I am also grateful to Scott Scheckleman and Dr. Martin Siderius for their very significant help and to Dr. Daniel Rouseff for serving on my thesis committee. Thanks also goes to the members of the NEAR-Lab, particularly Dr. Elizabeth Küsel, whose discussion, suggestions, and camaraderie were invaluable. Finally, I would like to acknowledge James Coleman at David Evans and associates for providing the acoustic data used, the Columbia River Crossing for providing geophysical measurements, and the Oregon Department of Transportation for funding this work. 


\section{Contents}

Abstract $\quad$ i

Acknowledgments

List of Tables $\quad$ vi

List of Figures vii

Chapter 1 Introduction 1

1.1 Executive Summary . . . . . . . . . . . . . . . 1

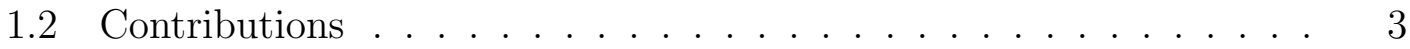

1.3 Impact Pile Driving . . . . . . . . . . . . . . . . . . 4

1.4 Thesis Work . . . . . . . . . . . . . . . . . . 6

$\begin{array}{llr}\text { Chapter } 2 & \text { Theory } & 10\end{array}$

2.1 The Propagation Model . . . . . . . . . . . . . . . 10

2.1.1 Shallow Water Sound Propagation . . . . . . . . . . . . 10

2.1.2 Parabolic Equation (PE) Propagation . . . . . . . . . . . 15

2.1.3 Frequency Synthesis . . . . . . . . . . . . . . . . 18 
2.2 Source Models . . . . . . . . . . . . . . . . . . . . . . . . . . . . 20

2.2 .1 Empirical Source Model . . . . . . . . . . . . . . . . . . 21

$2.2 .2 \quad$ FDTD Source Model . . . . . . . . . . . . . . . . . 27

2.3 Analysis Metrics . . . . . . . . . . . . . . . . 31

$\begin{array}{lll}\text { Chapter } 3 & \text { Experimental Data } & 34\end{array}$

3.1 Columbia River Enviornment _. . . . . . . . . . . . . . 34

3.2 The Geoacoustic Model . . . . . . . . . . . . . . . . . . . . . . 37

3.3 Experimental Data . . . . . . . . . . . . . . . . . 42

Chapter 4 Comparison to Observed Data $\quad 45$

4.1 Source Model Comparisons . . . . . . . . . . . . . . . . . . . . 45

4.2 PSD and SPL Comparison . . . . . . . . . . . . . 48

4.3 Sound Exposure Level Comparisons . . . . . . . . . . . . . . . 57

$\begin{array}{lll}\text { Chapter } 5 & \text { Applications } & 60\end{array}$

5.1 Contour Plots . . . . . . . . . . . . . . . . . 60

5.2 Bathymetry Effect $\ldots \ldots \ldots \ldots \ldots \ldots \ldots \ldots$

5.3 Sediment Layer Effect $\ldots \ldots \ldots \ldots 6$

$\begin{array}{lll}\text { Chapter } 6 & \text { Conclusion and Future Work } & 71\end{array}$

6.1 Conculsion . . . . . . . . . . . . . . . . 71

6.2 Future Work . . . . . . . . . . . . . . . . . . . . . 72 


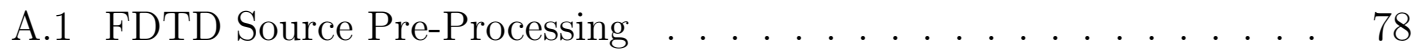

A.2 Broadband Propagation Model . . . . . . . . . . . . . 79

A.3 Empirical Source Post-Processing . . . . . . . . . . . . . 81

$\begin{array}{ll}\text { Appendix B RAM-PE Convergence } & 83\end{array}$

$\begin{array}{ll}\text { Appendix C } \text { Model Optimization } & 86\end{array}$

C.1 Parallel Computing . . . . . . . . . . . . . . . . . . 86

C.2 RAM-PE Source Code Modification . . . . . . . . . . . . 87 


\section{List of Tables}

3.1 Summary of Geoacoustic Parameters . . . . . . . . . . . . . . . . . 40

3.2 Pile Model Parameters . . . . . . . . . . . . . . . . . . . 41

4.1 Sound Exposure Level Summary . . . . . . . . . . . . . . 58

5.1 Parameters for Top Sediment Layer Comparison . . . . . . . . . . . 67 


\section{List of Figures}

1.1 Illustration of the modeling problem. The pile driving source is modeled as the deformation of the steel pile caused by the pile - hammer impact. Pile deformation is coupled into the propagation model, and propagated through the complex environment. . . . . . . . .

2.1 Illustration of the reflected and transmitted waves produced by an acoustic wave incident across a boundary between two media. Each boundary has distinct sound speeds $(c)$ and densities $(\rho)$. A portion of the wave is reflected at the angle of incidence $\left(\theta_{1}\right)$, and a portion is transmitted at the transmission angle, $\left(\theta_{2}\right) \ldots \ldots \ldots$

2.2 Possible multipath interactions between an arbitrary source and receiver. Illustrated are 1) the direct path, 2) the air-water interface reflected path, 3) the water-sediment reflected path, 4) the path transmitted through sediment 1 and reflected from sediment 2, and 5) the water-air, water-sediment, and water-air reflected path. The dotted arrows highlight the reflected or transmitted portion of the waveform that does not reach the receiver. . . . . . . . . . . 
2.3 The arrivals of the empirical source model. Panels (a), (b), and (c) show the bulge traveling through the pile and the emitted arrivals at progressively later times. $c_{p}$ is the speed of sound in the pile and $\psi_{w}$ is the arrival angle in the water, $\psi_{s}$ is the arrival angle in the sediment, and $\psi_{w}, s$ is the angle of the arrival that originated in the sediment and transitioned to the water. . . . . . . . . .

2.4 Example spectral weight function, $\gamma(f)$. This example was calculated from an empirically observed pile driving impact on pile B-1, taken at a distance of $10 \mathrm{~m} \ldots \ldots \ldots \ldots \ldots \ldots$

2.5 Isolation of the first arrival waveform. Panel (a) shows to observed waveform and panel (b) shows the observed waveform truncated according to equation $(2.21) \ldots \ldots \ldots \ldots \ldots$

2.6 Illustration of the FDTD Source Model, and the coupling into the propagation model. The cylindrical shells on the left represent the nodal particle velocity solutions of radius $a$ and height $d z$. The $\mathrm{N}$ particle velocity nodes are converted to $\mathrm{N}$ simple sources and convolved with the RAM-PE Green's functions solutions, represented by red dots at right. $\ldots \ldots \ldots \ldots \ldots \ldots$ 
3.1 Test Pile locations in the Columbia River. The areas of zero depth to the right of the pile driving sites demarcate the existing I5 span. Piles B1 and A1 are 24 inch diameter piles, and piles B2 and A3 are 48 inch diameter. The grayscale corresponds to river depth. . . . .

3.2 Acoustic observation sites in the Columbia River. Sites on the North and South riversides were used to observe the pile sites on the corresponding riversides. Measurements were taken at 10, 200, 400, and 800 meters from the pile driving site. . . . . . . . . . . .

3.3 The sediment layers used in the model. The arrows on the right of the figure indicate the variability in each sediment layer. The Troutdale Formation represents the acoustic basement and is effectively of infinite depth. . . . . . . . . . . . . . . .

3.4 Columbia River Bathymetry obtained from NOAA fathometer measurements. Bathymetry transects are taken directly from this data and used in the model. . . . . . . . . . . . . . . .

3.5 Example coring sample taken of the Troutdale Formation under the Columbia River. . . . . . . . . . . . . . . . . . .

3.6 Sound speed profiles for sites A and B. The lower sound speed regions at shallow depth correspond to the sand layer, the rapid transition corresponds to the narrow gravel layer, and the high sound speed region corresponds to the Troutdale Formation. . . . . . . . . . . 
3.7 Spectral weight functions used for modeling. Curves are derived from waveforms observed 10m for each pile. . . . . . . . . . . . . . 42

3.8 Example pile driving data record, which shows the SPL of several consecutive pile driving impacts. . . . . . . . . . . . . . .

3.9 Example ensemble averaged time domain waveform (A), sound exposure level (B), and PSD (C), for pile B1. The waveform and SEL are from the $10 \mathrm{~m}$ observation. PSD curves correspond to the four upstream measurement locations. . . . . . . . . . . . .

4.1 Short-range propagation still frames using the empirical source model to represent pile B1. The angled waves were emitted from the pile for a time period that included 4 arrivals. The black lines demarcate the sediment layer boundaries. The top layer is the water column, followed by sand, gravel and the Troutdale Formation, descending downward. . . . . . . . . . . . . . . .

4.2 Short-range propagation still frames using the FDTD source model to represent pile B1. The lines demarcate the sediment layer boundaries. The top layer is the water column, followed by sand, gravel and the Troutdale Formation, descending downward. . . . . . . . . 
4.3 PSD comparisons for pile B1. Panel A corresponds to the $10 \mathrm{~m} \mathrm{Ob-}$ servation site, B to $200 \mathrm{~m}, \mathrm{C}$ to $400 \mathrm{~m}$, D to $800 \mathrm{~m}$, and $\mathrm{E}$ to the $800 \mathrm{~m}$ site in the opposite direction. . . . . . . . . . . . . . . 50

4.4 PSD comparisons for pile B2. Panel A corresponds to the $10 \mathrm{~m} \mathrm{Ob-}$ servation site, B to $200 \mathrm{~m}, \mathrm{C}$ to $400 \mathrm{~m}$, D to $800 \mathrm{~m}$, and $\mathrm{E}$ to the $800 \mathrm{~m}$ site in the opposite direction. . . . . . . . . . . . . .

4.5 PSD comparisons for pile A1. Panel A corresponds to the $10 \mathrm{~m} \mathrm{Ob-}$ servation site, B to $200 \mathrm{~m}, \mathrm{C}$ to $400 \mathrm{~m}$, D to $800 \mathrm{~m}$, and $\mathrm{E}$ to the $800 \mathrm{~m}$ site in the opposite direction. . . . . . . . . . . . . . .

4.6 PSD comparisons for pile A3. Panel A corresponds to the $10 \mathrm{~m} \mathrm{Ob-}$ servation site, B to $200 \mathrm{~m}, \mathrm{C}$ to $400 \mathrm{~m}, \mathrm{D}$ to $800 \mathrm{~m}$, and $\mathrm{E}$ to the $800 \mathrm{~m}$ site in the opposite direction. . . . . . . . . . . . . . . .

4.7 SPL comparisons for pile B1. Panel A corresponds to the $10 \mathrm{~m}$ Observation site, B to $200 \mathrm{~m}, \mathrm{C}$ to $400 \mathrm{~m}$, D to $800 \mathrm{~m}$, and $\mathrm{E}$ to the $800 \mathrm{~m}$ site in the opposite direction. . . . . . . . . . . . . . . .

4.8 SPL comparisons for pile B2. Panel A corresponds to the $10 \mathrm{~m} \mathrm{Ob-}$ servation site, B to $200 \mathrm{~m}, \mathrm{C}$ to $400 \mathrm{~m}, \mathrm{D}$ to $800 \mathrm{~m}$, and $\mathrm{E}$ to the $800 \mathrm{~m}$ site in the opposite direction. . . . . . . . . . . . . . .

4.9 SPL comparisons for pile A1. Panel A corresponds to the $10 \mathrm{~m}$ Observation site, B to $200 \mathrm{~m}$, C to $400 \mathrm{~m}$, D to $800 \mathrm{~m}$, and $\mathrm{E}$ to the $800 \mathrm{~m}$ site in the opposite direction. . . . . . . . . . . . . . 
4.10 SPL comparisons for pile A3. Panel A corresponds to the $10 \mathrm{~m}$ Observation site, B to $200 \mathrm{~m}, \mathrm{C}$ to $400 \mathrm{~m}$, D to $800 \mathrm{~m}$, and E to the $800 \mathrm{~m}$ site in the opposite direction. . . . . . . . . . . . . . . . . 5

4.11 Sound exposure level summaries for each pile. The dotted lines correspond to results using the practical spreading model. The top line corresponds to an F factor of 5 and the bottom an $\mathrm{F}$ of 20 . The middle line is a fit to the data, and corresponds to an $\mathrm{F}$ of 10.5. . . 59

5.1 SEL contour plot about pile B1. The radial lines demarcate twodimensional simulation results, and the lines connecting radials connect points of equivalent SEL. . . . . . . . . . . . . .

5.2 SEL contour plot about pile A3. The radial lines demarcate twodimensional simulation results, and the lines connecting radials connect points of equivalent SEL. . . . . . . . . . . . . . .

5.3 Five bathymetry test cases, located near the I-5 Columbia River crossing. Test cases encompass the deep and shallow extremes, as well as the extremes for roughness and sloping bottoms found in the neighborhood of the construction site. . . . . . . . . . . . 64

5.4 Range dependent SEL at a depth of $3.5 \mathrm{~m}$, for each bathymetry test case. . . . . . . . . . . . . . . . . . 
5.5 Statistical threshold plot comparing the percent of the water column SEL above certain thresholds, for the different test cases. The region of comparison comprises the water column from range $100 \mathrm{~m}$ to $200 \mathrm{~m} \quad 65$

5.6 Range dependant SEL at 3.5m depth for multiple bedrock depths about pile B1. X demarcates the acoustic observations. Bedrock depths are relative to the simulation bathymetry. . . . . . . 6

5.7 Statistical threshold plot comparing the percentage of the water column above certain SELs, for several bedrock depths. Again, variations in a shallow bedrock layer have the greatest effect, and bedrocks below $25 \mathrm{~m}$ depth have little practical effect on the water column SEL. The region of consideration is the water column from range $100 \mathrm{~m}$ to

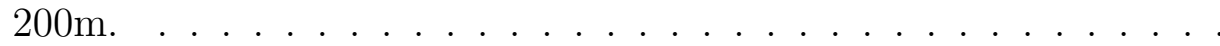

5.8 Range dependant SEL at 3.5m depth, for the top layer compositions in Table 5.1, about pile A3. X marks the observed SEL. . . . . . . .

5.9 Statistical threshold plot comparing the percentage of the water column about certain SEL, for each top layer composition. The area of

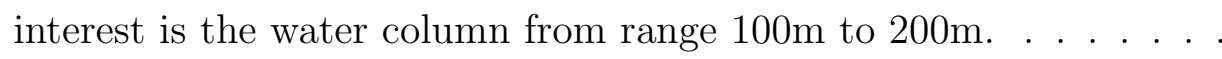

5.10 Geometrical explanation for the large SEL spike seen around $370 \mathrm{~m}$ in Figure 5.8. The propagation angle is assumed to change very little in the sediment due to the similar sound speeds. . . . . . . . . 70 
B.1 Illustration of the convergence testing method. Panel (a), (b), and (c) show the results of otherwise identical simulations computed using different spatial grids, where the grid sized increase from left to right. The curves at the bottom of each panel are the TL lifted from the upper part of the frame at a depth of $4.5 \mathrm{~m} . \ldots \ldots \ldots$ 


\section{Chapter 1}

\section{Introduction}

\section{$1.1 \quad$ Executive Summary}

The construction of bridges, piers, and other above-water structures involves impact pile driving which causes extremely high levels of underwater sound. These sound levels are known to have harmful physical and behavioral effects on marine wildlife [1]. Sound levels are difficult to predict due to the dynamic, time variant nature of the pile source, and the dependence on the complex and range-dependent underwater environments. Furthermore, while noise level prediction is a crucial component in the assessment and eventual mitigation of the environmental impacts caused by pile driving, it has previously received limited academic attention. Reinhall and Dahl [2] used a time-dependent source model coupled to broadband parabolic equation (PE) based solutions from the range-dependent acoustic model (RAM-PE [3]), that were capable of accounting for a range dependent environment and physics based sound propagation, but the analysis used only a basic sediment model and were compared to acoustic observations at only short ranges. Long range, broadband sound level predictions have been done using the RAM-PE [4], but only with simple sources in deep water environments, where bathymetry and sediment are less important. 
This thesis presents a computational model for the prediction and analysis of the underwater noise generated by impact pile driving that is suitable for use in shallow-water, range-dependent environments, over long ranges. Source models from recent technical literature are used, including both an empirical model from Reinhall and Dahl [2] and a finite difference time domain (FDTD) model from Shahab and Hastings [5]. Physics-based wave propagation is computed by broadband application of the RAM-PE, that uses a sediment model and bathymetry that are range dependent.

The computational model is applied to the Columbia River environment that lies between Portland, Oregon and Vancouver, Washington, which is advantageous due to the environmental monitoring and site characterization done in preparation for the construction of the new Interstate 5 bridge. Environmental monitoring included acoustic observations of test pile operations conducted by the consulting firm David Evans and Associates, which provides 20 observations over diverse range, sediment composition and pile size, suitablefor comparison with the model.

The model shows strong agreement with observations in both time and frequency domain metrics. Comparisons of power spectral density (PSD) and sound pressure level (SPL) show good agreement in roll-off and levels, and the cumulative sound exposure level (SEL) is predicted to within $4 \mathrm{~dB}$ at 22 of 25 comparisons, and at 17 of 17 of those comparisons made at sites located within $400 \mathrm{~m}$ of the pile driving site. The average discrepancy in SEL is $2.17 \mathrm{~dB}$ over all comparisons. 
The model is further applied to predict SEL over large areas of the Columbia River and study the effects of bathymetry and sediment configurations on sound levels in the environment. This analysis shows a significant dependence on bathymetry and sediment configurations that are not accounted for by previous modeling.

\subsection{Contributions}

- Developed a broadband wave propagation model using the convergent results of a modified RAM-PE code that incorporated range dependent sediment and bathymetry, suitable for propagating a pile driving source over long ranges in shallow water.

- Implemented the empirical source model of Reinhall and Dahl in the propagation model for multiple pile configurations.

- Coupled the results of the FDTD source model provided by the Georgia Institute of Technology into the propagation model by the formulation of a virtual simple source array.

- Compared simulation results to 20 acoustic observations of 24 and 48 inch piles at various locations on the north and south riversides of the Columbia River, in SPL, SEL, and PSD, and found good agreement.

- Studied the effects of variable bottom configurations and estimated SEL over large portions of the Columbia River. 


\subsection{Impact Pile Driving}

Bridge constructions, wind farm installation, and other forms of above water construction make use of impact pile driving, which causes extremely high sound levels in the surrounding environment. These sound levels are known to have harmful physical and behavioral effects on marine wildlife. Physical effects in fish include swim bladder rupture, torn tissue, bruising and hearing loss [1][6]. Behavioral effects are less understood, but can include altered migratory patterns and behaviors leading to increased predation [7]. Deleterious effects also extend to marine mammals, such as seals, and sediment bound marine life, such as fish eggs [8][9]. In all types of marine wildlife, specific harmful effects are highly species dependent, and are currently an area of major research.

The method of pile driving of greatest concern, and the focus of this work is impact pile driving, where repeated blows from heavy diesel or hydraulic hammers drive piles into the sediment. Impacts are delivered at a frequency between 60 and 15 strikes per minute and can produce extremely high sound levels in the surrounding underwater environment. Typical steel piles produce noise levels as high as $210 \mathrm{~dB}$ r: 1uPa and cast in steel-shell (CISS) piles can result in noise levels as high as $220 \mathrm{~dB}$ re: $1 \mu \mathrm{Pa}[10], 10 \mathrm{~m}$ from the pile driving source.

With the goal understanding and mitigating the environmental effects caused by pile driving, the study of pile driving has become a new area of academic interest. 
Existing research on pile driving has focused on effective observation methodologies [11], broad characterizations of pile driving sound [12], and methods of attenuating pile driving sound [13][14].

The prediction of underwater sound levels is important for the assessment of environmental impacts, but has received only limited academic attention. Regulatory agencies, such as the California and Oregon Departments of Transportation, currently employ the practical spreading model [15], which is a scaled logarithmic relationship that is limited to only predictions of absolute sound levels and is reliant upon fits to acoustic observations. Recently, Reinhall and Dahl presented an empirical model of the pile driving source that was based on array measurements of pile driving operations, and was propagated using the RAM-PE [2]. Good agreement was found between the model and observed data, but comparisons were only made out to $17 \mathrm{~m}$ from the pile driving source, where the effects of range dependent bathymetry and sediment are small. A long-range noise prediction was presented, but it only included a single frequency component and was not compared to acoustic observations. Long range broadband sound level predictions using PE propagation was done by Erbe et al. [4], but until now only a simple source has been used, and the studies correspond to a deep water environment. 


\subsection{Thesis Work}

In this thesis, a computational model is presented to predict and analyze the underwater sound produced by pile driving that addresses the challenges of long-range sound level prediction in shallow, inhomogeneous environments. This is challenging due to the dynamic, time-variant nature of the pile source and high dependence on the bathymetry and sediment. Density, attenuation and sound speeds in the various sediment layers can all affect the propagation of sound through the environment. This necessitates a suitable model of the pile source, a very well characterized environment, and a propagation model capable of accommodating sufficient sediment detail and physics based wave propagation.

The acoustic noise is predicted by incorporating three components (Figure 1.1): 1) a model of the pile driving source, i.e. the deformation of the pile caused by impact between hammer and pile, 2) coupling the pile driving source into a model of propagation, and 3) propagating the source through a complex environment.

The impact between hammer and pile produces a time-dependent deformation in the pile that results in the acoustic pressure waves in the environment. This model uses two contrasting models of the pile deformation, which have appeared in the recent technical literature. The first is an empirically based model of Reinhall and Dahl [2] that assumes most of the energy radiated results from an impulse bulge that rings in the pile. The second is an FDTD based model from Shahab 


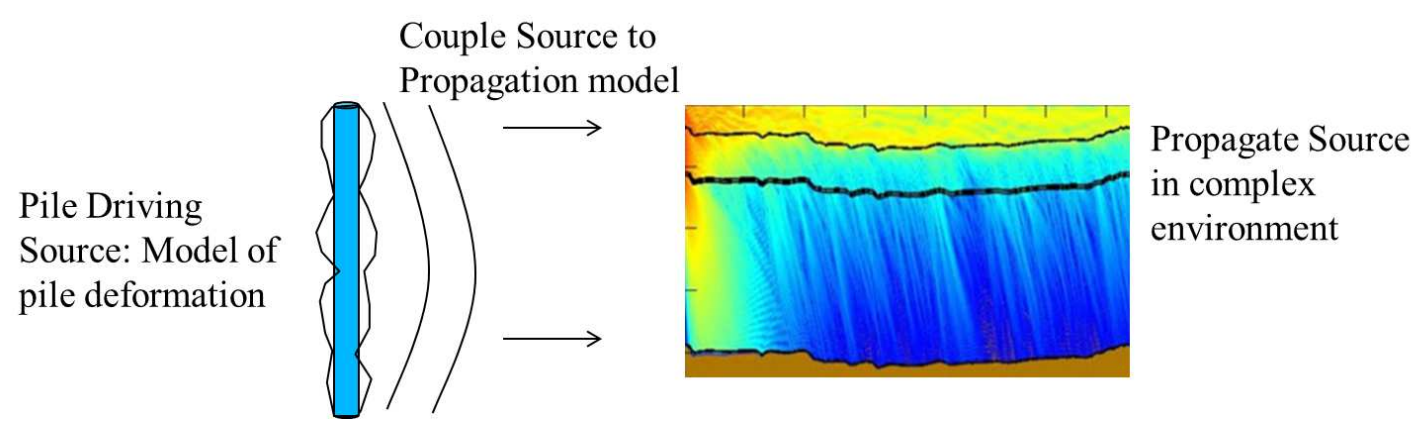

Figure 1.1: Illustration of the modeling problem. The pile driving source is modeled as the deformation of the steel pile caused by the pile - hammer impact. Pile deformation is coupled into the propagation model, and propagated through the complex environment.

and Hastings [5] that directly calculates the deformation of the pile based on the hammer impact waveform and a model of the pile and environment.

Modeling underwater acoustic propagation is a well-established academic field, with several mature methods available. Propagation methods include ray methods, wavenumber integration, normal modes, and parabolic equation $(\mathrm{PE})$ techniques [16]. Following the lead of initial efforts in pile driving noise prediction, PE techniques are used due to their suitability for range-dependent bathymetry and geoacoustic models as well as their accuracy in calculating low frequency solutions (most pile driving noise is present at low frequencies [12]). Calculation of PE solutions is performed using the range-dependent acoustic model (RAM-PE), a publicly available, standard PE propagation model developed by Collins at the Navel Research Laboratory [3]. Here, RAM-PE has been modified somewhat from the standard code to improve computational efficiency. 
The computational model is applied to the Columbia River environment that lies between Portland, OR and Vancouver, WA. This environment is advantageous due to the environmental monitoring and boring studies performed leading up to the proposed construction of a new Interstate 5 span in this area. The environmental monitoring consisted of acoustic observations of test pile observations in the north and south riversides performed by the consulting firm David Evans and Associates. Underwater acoustic waveforms due to pile driving of 24 and 48 inch piles were recorded at 5 locations each, at ranges from 10 to $800 \mathrm{~m}$, allowing close and long range comparisons between the model and data. Boring studies included descriptions of the sediment layers and compositions, laboratory sediment analysis and sound speed measurements, which were used in the derivation of the sediment geoacoustic model.

Beyond comparisons to acoustic data, the model was extended to predict SEL over large areas of the Columbia River and study the effects of bathymetry and sediment variations. Contour plots were generated using model solutions that were computed at several bearing angles from a common origin point, with areas of equivalent SEL connected. The effects of the bathymetry and sediment layers were studied by comparing identical simulations, with the parameters of study altered.

This thesis is structured as follows. Chapter 2 presents an introduction to shallow water sound propagation as well as the theoretical basis for PE modeling, broadband analysis, and the source models. The details of the Columbia River 
environment are presented in Chapter 3, along with the acoustic data used for model validation, and the geoacoustic model. Chapter 4 presents the comparison between simulation results and observed results and Chapter 5 presents the extended SEL predictions as well as an analysis of sediment and bathymetry configuration effects. Finally, Chapter 6 provides concluding remarks and suggests future work.

The appendices are concerned with the details of implementing the computational model, and are intended to be a suitable reference for further modeling work. Appendix A presents the model algorithm used to carry out the formulations in Chapter 2, Appendix B discusses RAM-PE convergence, which is critically important for accurate solutions, and Appendix $\mathrm{C}$ describes model optimization. 


\section{Chapter 2}

\section{Theory}

The intent of this chapter is to present the qualitative and mathematical content necessary for an understanding and intuition about the computational model. For clarity of presentation, propagation is discussed first. This includes an introduction to shallow water sound propagation and a discussion of the RAM-PE and parabolic equation techniques. Since RAM-PE produces a single frequency solution, the formulations used in the broadband synthesis of a time domain solution are presented. Next, descriptions and derivations of the empirical and FDTD source models are given. Finally, the time and frequency domain metrics used for comparison with empirical observations are defined. Due to the highly environment specific nature the of the geoacoustic model, the theory involved for this topic is held until Chap-

ter 3. For a detailed description of the method used to implement the formulation presented here, the reader is referred to Appendix A.

\subsection{The Propagation Model}

\subsubsection{Shallow Water Sound Propagation}

The propagation of acoustic waves in shallow water differs from other forms of acoustic propagation, such as in air or deep water, by the presence of boundaries 
that significantly affect sound propagation characteristics. These boundaries form an acoustic waveguide [16], which produces complex modal interference patterns in the water column. A detailed discussion of underwater propagation is beyond the scope of this thesis, so the reader is referred to Computational Ocean Acoustics [16]. This thesis will provide a discussion of the reflection and multipath dynamics that lead to the interference patterns, which should give a better intuitive understanding of the model. Finally, the sources of transmission loss (TL) and how they relate to the shallow water environment are discussed.

Boundaries to acoustic propagation are formed by the air-water interface as well as the sediment layers, all of which cause reflections and multipath interactions. The air water interface is considered to be a perfectly reflecting surface, with the entire incidence wave reflected at the angle of incidence. This is not the case for sediment reflections, where the wave is separated into reflected and transmitted parts [16], shown in Figure 2.1. The portions of the of the wave transmitted and reflected are proportional to the reflection coefficient,

$$
R=\frac{Z_{2}-Z_{1}}{Z_{2}+Z_{1}}
$$

and the transmission coefficient,

$$
T=\frac{2 Z_{2}}{Z_{2}+Z_{1}}
$$


Both coefficients are ratios of the impedances between the media. The impedance of the $i^{\text {th }}$ medium is,

$$
Z_{i}=\frac{\rho_{i} c_{i}}{\sin \theta_{i}}
$$

where $c_{1}$ and $\rho_{i}$ are the sound speed and density in the $i^{\text {th }}$ medium. The angle of reflection is equivalent to the incidence angle, $\theta_{1}$, and the angle of transmission is governed by Snell's Law,

$$
\frac{\omega}{c_{1}} \sin \theta_{1}=\frac{\omega}{c_{2}} \sin \theta_{2}
$$

where $\omega$ is the angular frequency of the incident wave.

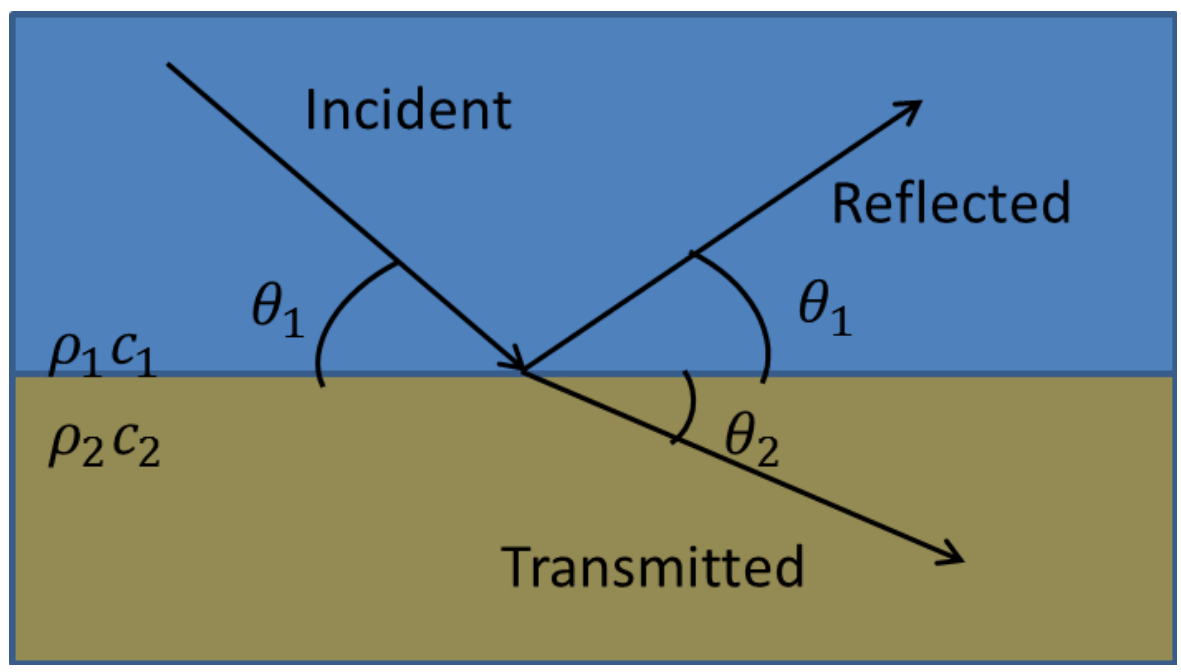

Figure 2.1: Illustration of the reflected and transmitted waves produced by an acoustic wave incident across a boundary between two media. Each boundary has distinct sound speeds $(c)$ and densities $(\rho)$. A portion of the wave is reflected at the angle of incidence $\left(\theta_{1}\right)$, and a portion is transmitted at the transmission angle, $\left(\theta_{2}\right)$. 
Reflections produce multipath effects, where waves arrive at the receiver from a common source that have traveled different path lengths due to reflections, illustrated in Figure 2.2. The difference in path length causes the wave fronts to differ in phase, causing constructive and destructive interference. Waves that are in phase will add constructively, while waves that are half-wavelengths out of phase will become vanishingly small.

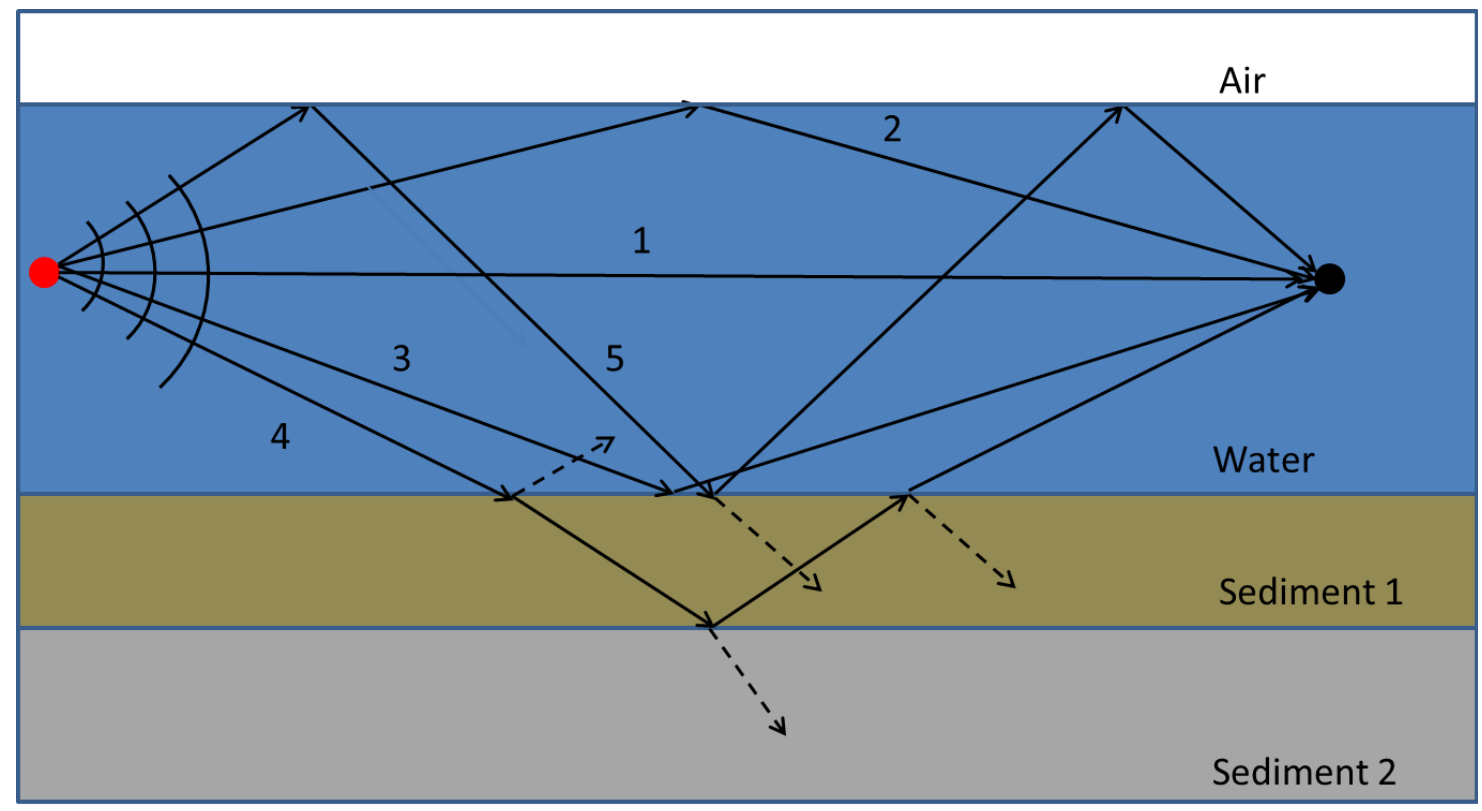

Figure 2.2: Possible multipath interactions between an arbitrary source and receiver. Illustrated are 1) the direct path, 2) the air-water interface reflected path, $3)$ the water-sediment reflected path, 4) the path transmitted through sediment 1 and reflected from sediment 2, and 5) the water-air, water-sediment, and water-air reflected path. The dotted arrows highlight the reflected or transmitted portion of the waveform that does not reach the receiver.

TL results from two factors: spreading loss and attenuation. If an acoustic source is able to propagate freely into space, the acoustic energy will expand into the area of a sphere, and the source will experience spherical spreading. Conversely, 
a source that is present between two perfectly reflecting parallel planes will only expand into the area of a cylinder and experience cylindrical spreading. In spherical spreading, TL is proportional to the inverse of the range squared, and in cylindrical spreading, it is proportional to only the inverse of the range.

In the shallow water case, the sound source is bounded by a perfectly reflecting plane (air-water) and lossy, reflective sediment layers. While sound attenuation in water is commonly considered to be negligible, the attenuation in the sediment is not, and interactions with the sediment result in losses. For example, in Figure 2.2 before path 4 reaches the receiver, it has experienced losses from transitioning to sediment 1 , traveling through sediment 1 , reflecting with sediment 2 , and transitioning again to the water column. This is critical for an intuitive understanding of the sound attenuation predicted by the model. For example, shallower bathymetry results in increased reflections on the sediment-water interface and therefor greater loss.

An attempt has been made to amalgamate the various sources of TL into a simple model, known as the practical spreading model [15], which is currently used by regulatory agencies such as the California and Oregon Departments of Transportation. This model simply computes the sound level by subtracting the scaled logarithm of range from an assumed source level,

$$
L V L=S R C L V L-F \log r .
$$


Here $L V L$ is the sound level at range $r, S R C L V L$ is the assumed source level, and $F$ is an attenuation factor that is allowed to vary from 5 to 30 . This method has a number of problems. First, it assumes an environment that is homogeneous and axisymmetric, when in reality variations in sediment composition and bathymetry can significantly affect sound levels. The second problem is that $S R C L V L$ and $F$ cannot be trivially obtained, and must be determined by fitting acoustic data at several ranges.

\subsubsection{Parabolic Equation (PE) Propagation}

PE propagation was chosen because the method is well suited to calculate propagation in range-dependent and arbitrarily complex environments. That is, it computes physics based wave propagation, including the interactions described above, for sediment layers and bathymetry that vary in range. Furthermore, the method was chosen because it is suitable for low frequency calculations, (below approximately $3000 \mathrm{~Hz}$ ) where most pile driving energy is contained [12]. Computation of PE solutions was done using a mature and publicly available PE code, the RAM-PE [3]. The RAM-PE was originally developed for application to problems in sonar, and calculates the frequency dependent wave propagation through a two-dimensional geometry. RAM-PE calculates solutions by solving the two-dimensional acoustic wave equation, 


$$
\frac{\delta^{2} p}{\delta r^{2}}+\rho \frac{\delta}{\delta z}\left(\frac{1}{\rho} \frac{\delta p}{\delta z}\right)+k^{2} p=0
$$

where $p$ is the acoustic pressure, $\rho$ is the density, $z$ is the receiver depth, $r$ is the receiver range, and $k$ is the wave number, $k=\frac{2 \pi}{\lambda}$, where $\lambda$ is the wavelength in the medium at the current range and depth. The solutions to this expression are Green's functions, which solve (2.6) for a given set of boundary conditions and medium inhomogeneities. The Greens function is two-dimensional and frequency dependent, $G(r, z, f)$.

In the absence of environmental inhomogeneities and boundaries, (2.6) is solved by the free space point source,

$$
G(r, z, f)=\frac{\exp \left(-i k r\left(r^{2}+z^{2}\right)^{2}\right)}{\left(r^{2}+z^{2}\right)^{2}}
$$

The RAM-PE simplifies the wave equation by factoring (2.6) into a parabolic form, assuming forward energy dominates, and calculating solutions to the forward component of the wave equation,

$$
\frac{\delta \rho}{\delta r}=i k_{0}(1+X)^{\frac{1}{2}} p
$$

Here $k_{0}=\frac{\omega}{\nu}$, where $\omega$ is the angular simulation frequency and $\nu$ is the representative phase speed. The operator $X$ assures that (2.8) is satisfied for the given 
environmental boundary conditions and inhomogeneities,

$$
X=k_{0}^{-2}\left(\rho \frac{\delta}{\delta z} \frac{1}{\rho} \frac{\delta}{\delta z}+k^{2}-k_{0}^{2}\right)
$$

RAM solves (2.8) using a recursive relationship that calculates range dependent solutions based on a q term rational approximation,

$$
G(r+\Delta r, z, f)=e^{i k \Delta r} \prod_{q=1}^{Q} \frac{1+C_{q, Q} X}{1+B_{q, Q} X} \rho(r, z)
$$

where $C_{q, Q}$ and $B_{q, Q}$ are Padé series coefficients.

The initial fields (those fields at the first range step) are calculated using the self-starter, which calculates a particular solution to (2.6),

$$
\frac{\delta \rho}{\delta r^{2}}+\rho \frac{\delta}{\delta z}\left(\frac{1}{\rho} \frac{\delta \rho}{\delta z}\right)+k^{2} \rho=2 i \delta\left(z-z_{0}\right)
$$

where $z_{0}$ is the source depth. It is solved by,

$$
G\left(r_{0}, z, f\right)=\frac{\exp \left(i k_{0} r_{0}(1+X)^{1 / 2}\right)}{k_{0}^{1 / 2}(1+X)^{1 / 4}} \delta\left(z-z_{0}\right)
$$

The resulting two-dimensional greens function solutions give the complex pressure fields in the specified environment, as produced by a $1 \mu P a$ source. The implementation and problem specific optimization of the RAM-PE are discussed in the appendices. 


\subsubsection{Frequency Synthesis}

While the propagation model calculates a single frequency solution, broadband spectral and time domain analysis are of primary concern. Computing Greens function solutions over a broad band produces a two-dimensional frequency domain solution demarcated, $S(r, z, f)$. A time domain solution was obtained by synthesis of the frequency domain solution and carried out using the discrete Fourier transform (DFT),

$$
s_{n}(r, z, \eta)=\frac{2}{N T} \operatorname{Re}\left\{\sum_{N} S_{n}(r, z, n) e^{-2 n \frac{\eta}{N} n}\right\}
$$

where $\mathrm{N}$ is the total number of points in the transform, $\mathrm{n}$ is the nth frequency domain sample, and $\eta$ is the $\eta^{\text {th }}$ time domain sample. Since the negative frequency components are not calculated, the solution is multiplied by a factor of two, and the real part of the solution is taken. The resolution in the time domain is,

$$
\Delta t=\frac{1}{N \Delta f}
$$

where $\Delta f$ is the frequency resolution. The time axis corresponding to the $\eta^{t h}$ bin is,

$$
t=[\Delta t, 2 \Delta t, 3 \Delta t, \ldots, N \Delta t]
$$


Computational efficiency is improved by selecting broadband parameters that accommodate the requirements of a robust model, but do not require more calculations than are needed. To this end, frequency domain parameters are chosen to accommodate the necessary time domain period as well as the relatively low frequency limitations of PE modeling.

The frequency resolution was chosen to create a time period sufficient for time domain propagation to the extent of the simulation range. That is, sufficient time so that the source function solution can propagate the length of the simulation area. This is based on the water column waveguide group velocity,

$$
v_{g}=c_{w} \sqrt{1-\left(\frac{\omega_{0}}{\omega}\right)^{2}},
$$

where $\omega$ is the maximum simulation angular frequency and $\omega_{0}$ is the waveguide cutoff frequency. The cutoff frequency for the first mode is,

$$
\omega_{0}=2 \pi \frac{c_{w}}{2 D},
$$

where $c_{w}$ is the speed of sound in water and $D$ is the average depth of the water column. The necessary frequency resolution is,

$$
\Delta f=\frac{v_{g}}{R_{\max }},
$$


where $R_{\max }$ is the maximum range of the simulation. For a maximum observation range of $800 \mathrm{~m}$, the frequency step is approximately $1.5 \mathrm{~Hz}$.

The bandwidth is chosen to provide the greatest computational efficiency while still capturing most of the signal energy. This is done by spectral analysis of a close range, empirically observed, pile driving waveform. An appropriate bandwidth is selected by comparing the sum of the energy spectral density in the full spectrum of the close range observed signal, to that of the observed signal after a low pass filter has been applied. The percentage of the total signal energy in the truncated simulation is given by the fraction of the summed Energy Spectral Density (ESD),

$$
B=\frac{\sum E S D_{\text {Obs }}}{\sum E S D_{\text {Truncated }}},
$$

where $E S D_{O b s}$ is the ESD summed over all bins in the observed measurement, $E S D_{\text {Truncated }}$ is the bandwidth truncated ESD, and $B$ is the ratio between the two. For the 24 inch piles in this work, a maximum frequency of $2600 \mathrm{~Hz}$ contained 97 percent of the total signal energy, while for the 48 inch piles; a maximum frequency of only $2100 \mathrm{~Hz}$ was needed to capture the same percentage of energy.

\subsection{Source Models}

The impact between the hammer and pile causes a deformation in the pile material, which results in the initial pressure fields in the environment. The empirical and 
FDTD source models presented below provide contrasting models of this deformation, which will be quantified in Chapter 4. In both cases, the source functions are coupled into the propagation model to produce a two-dimensional broadband simulation. These Green's function solutions are demarcated $S_{e m p}(r, z, f)$ for the empirical source model, and $S_{F D T D}(r, z, f)$ for the FDTD source model. Results using both source models can be processed identically, and the subscript is only kept to specify details of the simulation.

\subsubsection{Empirical Source Model}

The empirical source model of Reinhall and Dahl [2] assumes the vast majority of the energy radiated into the environment results from an impulse bulge traveling through the pile. This bulge travels at the speed of sound in steel, reflects at the ends of the pile, and is attenuated at the reflection between the pile and sediment. Since the speed of sound in the steel pile $\left(c_{p}=5100 \frac{\mathrm{m}}{\mathrm{s}}\right)$ is much greater than that in the environment $\left(c_{w}=1447 \frac{m}{s}\right)$, energy is radiated in conical arrivals of incidence angle,

$$
\psi=\arcsin \left(\frac{c_{p}}{c_{s}}\right),
$$

where $c_{p}$ is the speed of sound in the pile and $c_{s}$ is the speed of sound in the sediment. Also, $c_{w}$, the sound speed in water, is substituted in (2.20) for $c_{w}$ if the 
bulge is currently in the water column rather than then sediment. At each instance of the bulge traversing the pile, a conical arrival is generated, shown in Figure 2.3. Panel (a) shows the first arrival, shortly after the hammer impact. Panel (b) shows the first arrival in the sediment, with conical waves traveling at slightly different angles, based in the difference between water and sediment sound speed. Also, the beginning of the second arrival can be seen, as the bulge has reflected at the bottom of the pile and is now traveling up the pile. Panel (c) shows the full second arrival, with the conical waves described in Panel (b), as well as a small wave at a third angle, which originated in the sediment and has transitioned into the water. The bulge continues to reflect at the top and bottom of the pile and produce similar arrivals, until it has been completely attenuated. The bulge is considered to be of uniform amplitude while traversing the pile, and is attenuated at the pile-sediment reflection. 

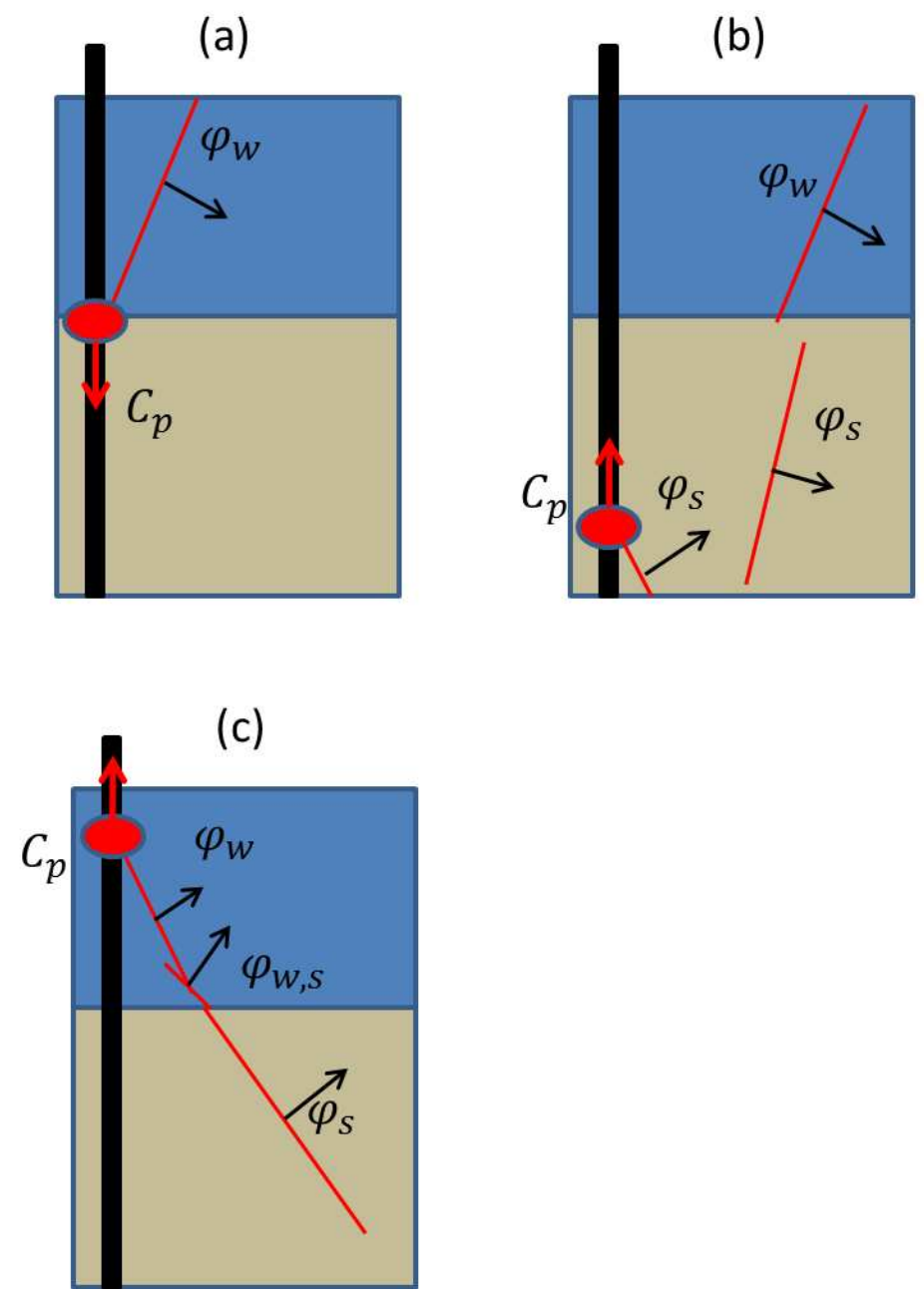

Figure 2.3: The arrivals of the empirical source model. Panels (a), (b), and (c) show the bulge traveling through the pile and the emitted arrivals at progressively later times. $c_{p}$ is the speed of sound in the pile and $\psi_{w}$ is the arrival angle in the water, $\psi_{s}$ is the arrival angle in the sediment, and $\psi_{w}, s$ is the angle of the arrival that originated in the sediment and transitioned to the water.

The empirical source model is named as such because it is dependent upon a close-range acoustic measurement. The acoustic measurement is used in two ways: by the application of a spectral weighting function $\gamma(f)$ and an energy matching offset $C$. 
The spectral weighting function, $\gamma(f)$ is defined as the normalized Fourier transform magnitude of the first arrival from a close-range observed waveform. Figure 2.4 shows an example spectral weight function, originating from pile B-1. It is used to weight the magnitude of each frequency dependent simulation such that the simulated and observed spectra match at close range.

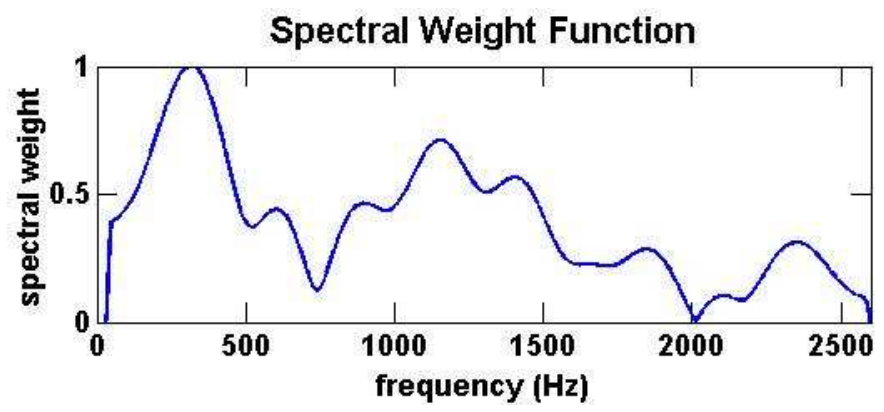

Figure 2.4: Example spectral weight function, $\gamma(f)$. This example was calculated from an empirically observed pile driving impact on pile B-1, taken at a distance of $10 \mathrm{~m}$.

The value of $\gamma(f)$ is calculated as follows. The first arrival is isolated by calculating the time that the second arrival requires to reach the hydrophone, after the start of the signal. All samples after this time are discarded. By a geometrical argument, the time of the first arrival after the start of this signal is,

$$
t_{1,2}=\frac{s\left(p_{w l}-z_{r}\right)}{c_{p}},
$$

where $p_{w l}$ is the wetted length of the pile, and $z_{r}$ is the depth of the receiver. The isolation of the first arrival is shown in Figure 2.5. The observed waveform in panel (a) is truncated to the first arrival in panel (b) by discarding all points after the 
time defined in equation (2.21).

(a)

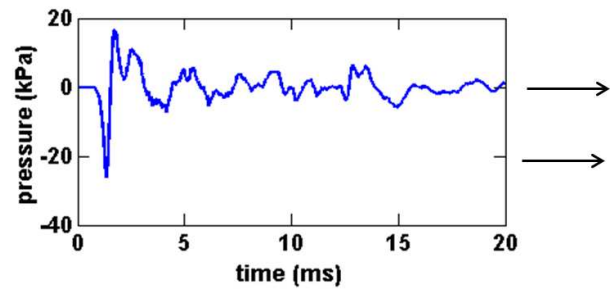

(b)

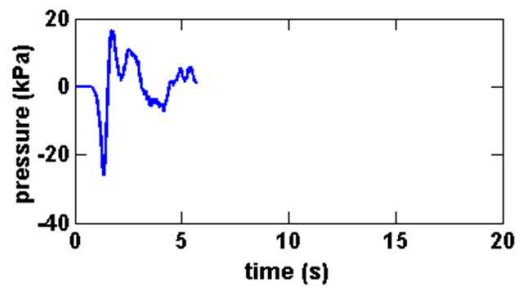

Figure 2.5: Isolation of the first arrival waveform. Panel (a) shows to observed waveform and panel (b) shows the observed waveform truncated according to equation (2.21).

The first arrival is then converted to the frequency domain using the DFT, normalized, and truncated to the bandwidth of the numerical simulation. Finally, a gentle 0.015 factor Tukey window [17] was applied to reduce any ringing effects in the time domain synthesis.

The source was implemented by forming each $m^{\text {th }}$ conical arrival separately as a steered array of point sources solutions, invoking Huygens' principle. The expression for the $j^{\text {th }}$ depth point source of the empirical source model is,

$$
s_{\text {emp }, m, j}(r, z, f)=G(r, z, f) \gamma(f) e^{-i 2 \pi f \tau_{m, j}},
$$

where $G(r, z, f)$ is a broadband point source solution, which is the component computed by the RAM-PE at each frequency. The emp subscript denotes the empirical source model. The exponential term is a depth dependent time delay of $\tau_{m, j}$ that steers the arrival to the proper incidence angle, $(2.20)$ by the shift theorem 
[17]. This is similar to steering a beamformer array. The time delay is the time required for the bulge to reach the $j^{\text {th }}$ depth point source on the pile and depends on the dimensions of the specific pile modeled. The delays $\tau_{m, j}$ for the first four $m^{\text {th }}$ arrivals at the $j^{\text {th }}$ source depth are,

$$
\tau_{1, j}=\frac{c_{p}}{d_{j}}, \quad \tau_{2, j}=\frac{2 p_{w l}-d_{j}}{c_{p}}, \quad \tau_{3, j}=\frac{2 p_{w l}+d_{j}}{c_{p}}, \quad \tau_{4, j}=\frac{4 p_{w l}-d_{j}}{c_{p}} .
$$

where $d_{j}$ is the depth of the $j^{\text {th }}$ point source.

Summing over all source depths results in the conical wave of the $m^{\text {th }}$ arrival,

$$
S_{e m p, m}(r, z, f)=\frac{1}{J} \sum_{j=1}^{J} s_{e m p, m, j}(r, z, f)
$$

where $J$ is the total number of point sources. The full simulation is found by summing over all arrivals, accounting for bulge attenuation and applying the energy matching constant.

$$
S_{e m p}(r, z, f)=C \sum_{m=1}^{M}(-1)^{m+1} K_{m} S_{e m p, m}(r, z, f)
$$

Here $C$ is the energy matching constant, and $K_{m}$ is a constant parameter which models the attenuation of the bulge at each reflection, 


$$
K_{m}=\left\{\begin{array}{l}
\kappa^{m-2}, \text { if } m=1,3,5, \ldots \\
\kappa^{m-1}, \text { if } m=2,4,6, \ldots
\end{array}\right.
$$

where $\kappa$ is a real number between zero and one, and is equal to the amplitude ratio of the first and third arrival peaks. The energy matching constant $C$ is chosen such that the sum of the ESD of the simulated spectrum matches the sum of the ESD of the observed signal, at the observation point, over the simulation bandwidth. It is a function of the observed and simulated signals,

$$
C=\frac{\sum E S D_{\text {Observed }}}{\sum E S D_{\text {Simulated }}} .
$$

\subsubsection{FDTD Source Model}

Under the ODOT sponsored program that supported this thesis, Shahab and Hastings at the Georgia Institute of Technology (GTech) worked under subcontract with the NEAR-Lab to develop a rigorous and purely computational model of the pile deformation. Their work culminated in the development of a FDTD model of deformation at discrete elements along the pile surface [5]. The model uses several parameters including the full geoacoustic model, the pile material and dimensions, the cap placed between the pile and hammer, and the force waveform between the hammer and pile. The model solves the equations of motion of a cylindrical shell, 
with the boundary condition that the shell is partially enveloped in the environment of interest [18], using an FDTD [19][20][21] method. Solutions were provided by the GTech.

The coupling of the FDTD model into the propagation model is described in Figure 2.6. The FDTD model calculates pile deformation as the particle velocity of $\mathrm{N}$ discrete cylindrical shells. Since the RAM-PE calculates the solution to a spherically radiating point source, the cylindrical particle velocity solutions are coupled into the propagation model by formulation of the simple source pressure field [22], which is convolved with the PE model point source array [22].

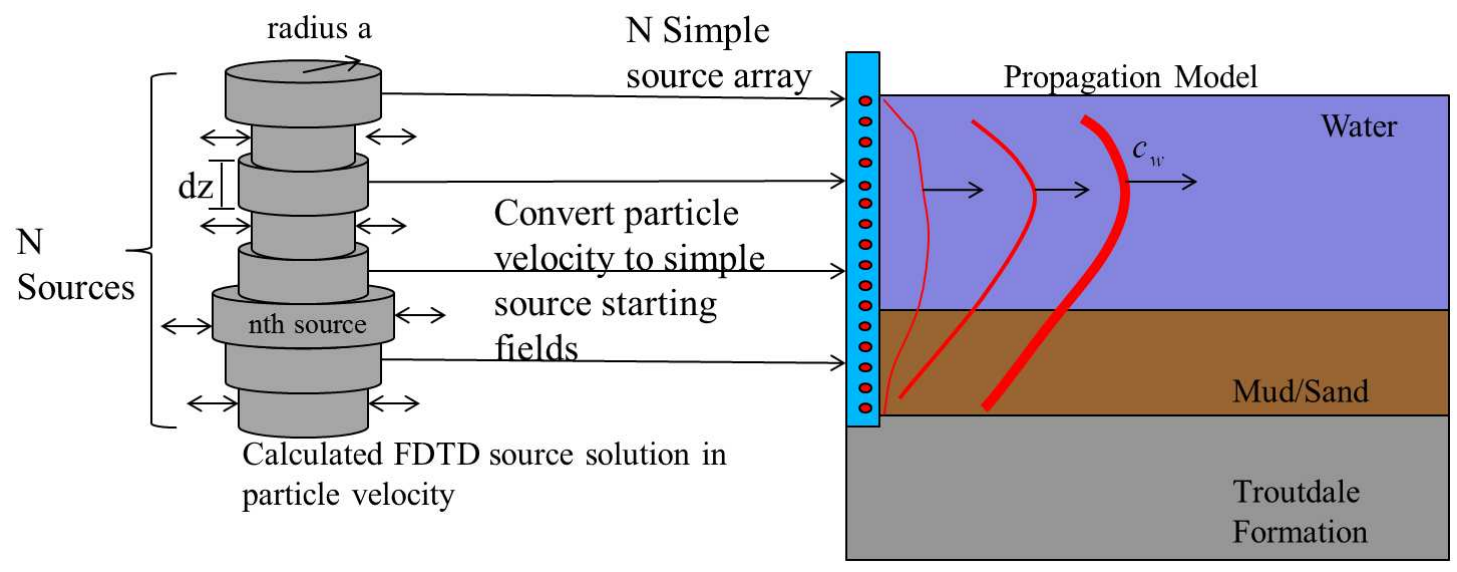

Figure 2.6: Illustration of the FDTD Source Model, and the coupling into the propagation model. The cylindrical shells on the left represent the nodal particle velocity solutions of radius $a$ and height $d z$. The $\mathrm{N}$ particle velocity nodes are converted to $\mathrm{N}$ simple sources and convolved with the RAM-PE Green's functions solutions, represented by red dots at right.

The pressure field of the $j^{t h}$ simple source is, 


$$
p_{j}(r, t, f)=\zeta_{j}\left(\frac{Q}{4 \pi a \xi}\right) e^{i[\omega t-k(\xi-a)]}
$$

where $a$ is the simple source radius, $\xi$ is the distance from the origin, $\zeta$ is the specific acoustic impedance of a sphere,

$$
\zeta_{j}(f)=\rho_{j} c_{j} \frac{[k(f) \xi]^{2}}{1+[k(f) r]^{2}}+i \rho_{j} c_{j} \frac{k(f) \xi}{1+[k(f) \xi]^{2}}
$$

As with the empirical source model, $j$ indexes the $j^{\text {th }}$ source depth, $\rho_{j}$ and $c_{j}$ are the density and sound speed surrounding the $j^{\text {th }}$ point source and $Q_{j}$ is the source strength of a moving cylindrical shell,

$$
Q_{j}=2 \pi a U_{j}(f) d z
$$

Here, $d z$ is the node depth spacing, and $U_{j}(f)$ is the discrete Fourier transform of the time domain particle velocity. Combining (2.28) and (2.30) and imposing the conditions that $t=0$ and $\xi=a$ results in the starting pressure fields for a single node,

$$
p_{j}(f)=\zeta_{j}(f) \frac{U_{j}(f) d z}{2 a} .
$$

The starting pressure field is propagated by convolution with the specific Green's function, calculated by RAM, 


$$
P_{j}(r, z, f)=p_{j}(f) G_{j}(r, z, f) .
$$

The total Green's function is the sum of the propagated simple sources,

$$
S_{F D T D}(r, z, f)=\sum_{j=1}^{J} P_{j}(r, z, f),
$$

where the FDTD subscript denotes the FDTD source model.

The simple source formulation derived above is dependent upon the pressure at the uniformly vibrating surface of the modeled source being approximately equal to the pressure that would be produced at the surface of an identically vibrating sphere. For arbitrary sources, this assumption requires that $k a<<1$. While this condition is not satisfied at the upper frequencies of the model, it was shown to be valid for the cylindrical source in question.

The pressure field of an arbitrary source is the particle velocity $u$ multiplied by the acoustic impedance $\zeta$,

$$
p=u \zeta,
$$

Therefore, for identical particle velocities, the ratio of pressure is the ratio of the specific acoustic impedances. Using (2.34) and the specific acoustic impedance of a cylinder, 


$$
\zeta_{c y l i n d e r}=\frac{i \rho_{0} c H_{0}^{(2)}(k r)}{H_{1}^{(2)}(k r)}
$$

where $H_{0}$ and $H_{1}$ are Hankel functions of the first and second kind, the magnitude of the pressure ratio at the surface of the cylindrical shell and maximum frequency is given by,

$$
\frac{\left|p_{\text {sphere }}\right|}{\left|p_{\text {cylinder }}\right|}=\frac{\zeta_{\text {sphere }}}{\zeta_{\text {cylinder }}}
$$

When evaluated at $r=0.609 \mathrm{~m}$ and $f=2600 \mathrm{~Hz}$, the ratio defined in (2.36) is 0.9952. Thus the approximation is accurate to less than one part in one hundred, and should be reasonable.

\subsection{Analysis Metrics}

The total Green's function propagation solutions for both source models, $S_{\text {emp }}(r, z, f)$ and $S_{F D T D}(r, z, f)$, define the broadband complex pressure at each point in the simulation area. These results can be directly compared with the observed data using several frequency and time domain metrics, defined in this section.

Frequency domain analysis is performed by examination of the Energy Spectral Density (ESD),

$$
E S D=S(r, z, f)^{2}
$$


and Power Spectral Density (PSD),

$$
P S D=\frac{v}{L} \sum_{l=0}^{L-1} P_{l}(f),
$$

where $P_{l}(f)$ is the $l^{\text {th }}$ windowed periodogram and $L$ is the total number of periodograms. Periodograms are obtained by first dividing the time domain waveform into L equal segments that overlap. The segments are then windowed and transformed to the frequency domain. The periodograms are calculated by computing the ESD of the windowed and transformed segments. The correction factor $v$ is a constant that corrects for the energy lost by windowing,

$$
v=\frac{1}{G} \sum_{g=0}^{G-1} w^{2}(g),
$$

where $w(g)$ is the $g^{\text {th }}$ window function bin, and $G$ is the total number of points in the window. Note that for PSD analysis of the simulated data, a time domain waveform must be synthesized for application of the PSD algorithm.

In the time domain, data was analyzed in terms of amplitude,

$$
A=\|s(r, z, t)\|^{2},
$$

and Sound Pressure Level (SPL), 


$$
S P L=10 \log _{10}(A),
$$

where $S P L$ is relative to $1 \mu P a$ and has units of $d B$ relative to $1 \mu P a$, and Sound Exposure Level (SEL),

$$
S E L=10 \log _{10}\left(\sum_{k=1}^{K} A_{k} \Delta t\right),
$$

which has units of $d B$ relative to $1 \mu P a^{2} s$. 


\section{Chapter 3}

\section{Experimental Data}

This work focuses on the shallow water environment of the Columbia River, between Portland, OR and Vancouver, WA, in the area of the proposed new Interstate 5 span over the Columbia River. The construction of the new I5 span is a high profile construction project that will include extensive pile driving, over long periods of time. This chapter describes the Columbia River environment to which the model was applied in detail, the observed data used for comparisons with simulated results, and the geoacoustic model derived based on the environment. The specific parameters used in the model and the spectral weight functions for each of the pile are also given.

\subsection{Columbia River Enviornment}

The Columbia River between Portland, OR and Vancouver, WA is a highly advantageous area for the study of pile driving due to the extensive environmental monitoring and survey work done in preparation for the construction of the proposed I5 span. Environmental monitoring of test pile operations was done in February, 2011. In these tests, steel piles of 24 and 48 inch diameters were driven into both the north (Pile Site B) and south (Pile Site A) riversides, shown in Figure 3.1. On the north riverside, the 24 inch and 48 inch piles are labeled B-1 and B-2 
respectively, and on the south riverside, they are labeled A-1 and A-3. Acoustic observations were taken by the consulting firm David Evans and Associates of each pile at 5 locations, ranging from 10 to 800 meters. This provided 20 high quality measurements, spanning diverse ranges, variations in bathymetry, and pile types, for model comparisons (Figure 3.2).

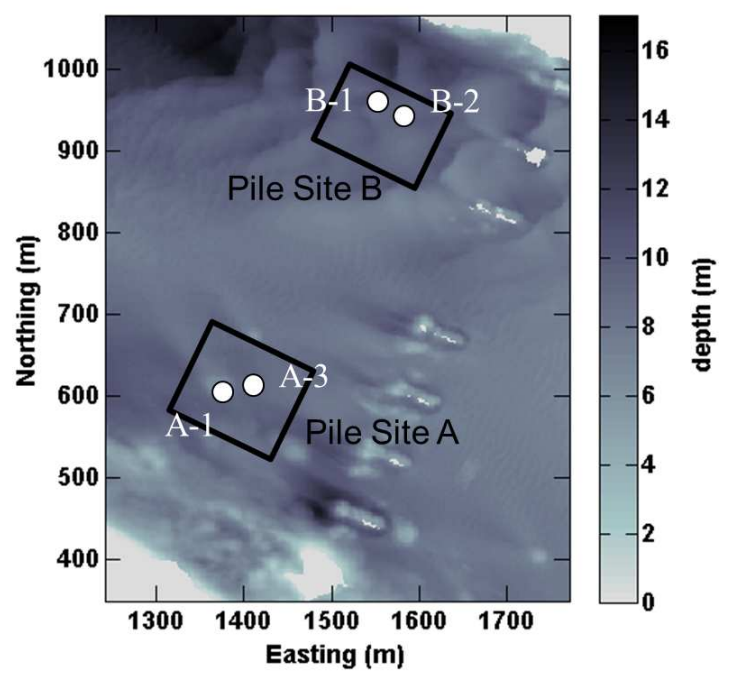

Figure 3.1: Test Pile locations in the Columbia River. The areas of zero depth to the right of the pile driving sites demarcate the existing I5 span. Piles B1 and A1 are 24 inch diameter piles, and piles B2 and A3 are 48 inch diameter. The grayscale corresponds to river depth. 

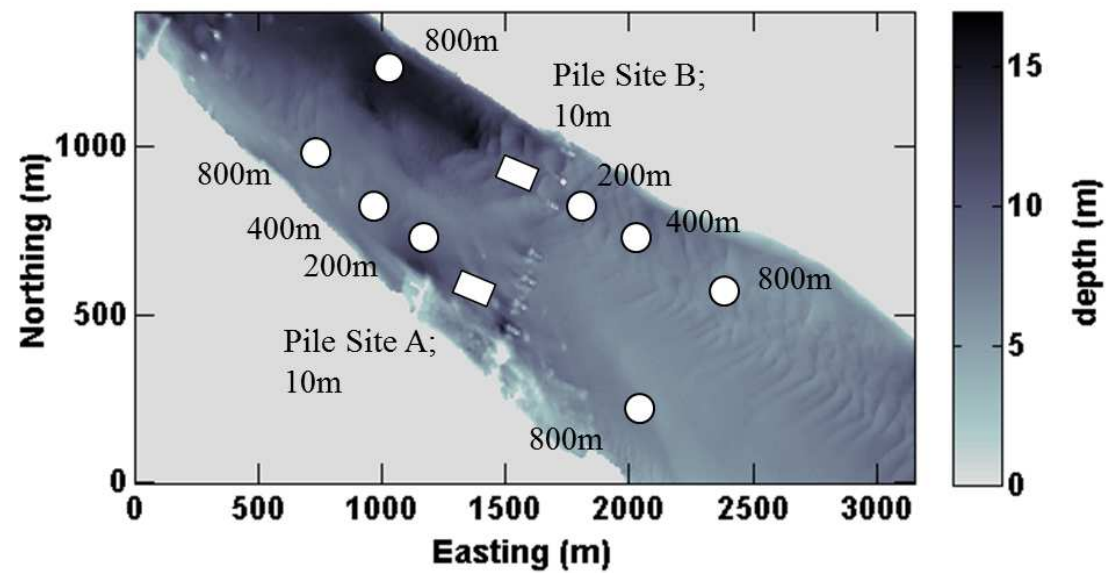

Figure 3.2: Acoustic observation sites in the Columbia River. Sites on the North and South riversides were used to observe the pile sites on the corresponding riversides. Measurements were taken at 10, 200, 400, and 800 meters from the pile driving site.

Survey work includes detailed bathymetry measurements as well as boring and laboratory sediment analysis at several locations [23][24]. The bathymetry shows a shallow, inhomogeneous river environment, with main channel depths from 6 to 17 meters. The boring records indicate three main sediment layers (Figure 3.3): a top layer of medium grained sand, a thin gravel layer, and a dense cobblestone bedrock layer, known as the Troutdale Formation. Measurements of grain size, porosity and sound speed were taken for each of these layers at multiple locations. Also of interest is the depth of the Troutdale formation, which varies substantially across the width of the river. At pile site B, the Troutdale Formation is as shallow as $12.5 \mathrm{~m}$ below the mud line, whereas at pile site $\mathrm{A}$, it is as deep as $60 \mathrm{~m}$. This allows the evaluation of the effects of this dense, highly reflective layer. 


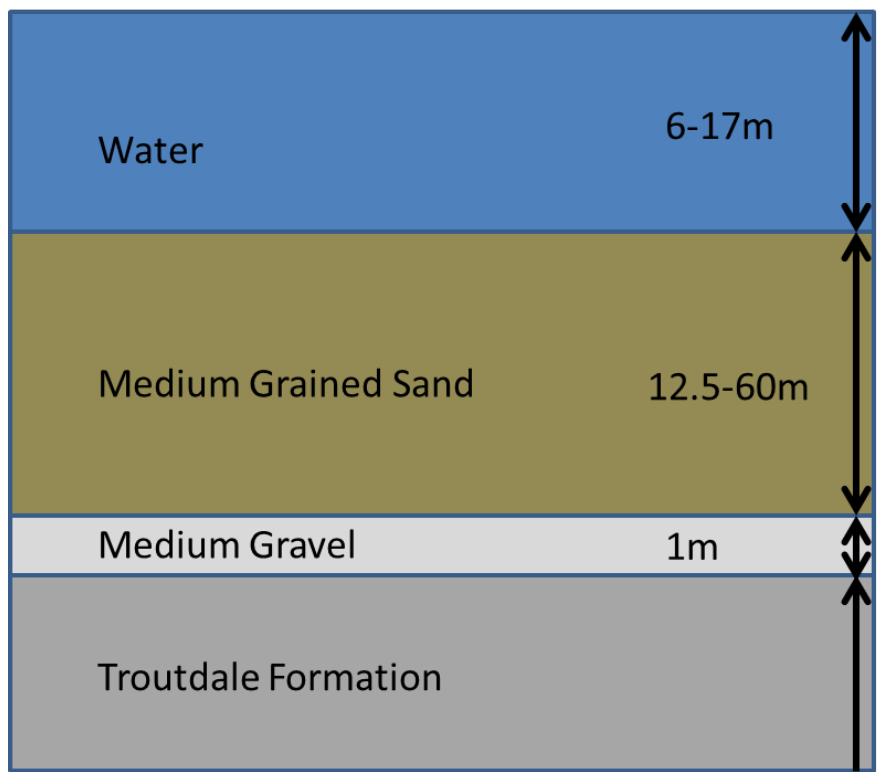

Figure 3.3: The sediment layers used in the model. The arrows on the right of the figure indicate the variability in each sediment layer. The Troutdale Formation represents the acoustic basement and is effectively of infinite depth.

\subsection{The Geoacoustic Model}

PE modeling allows the use of range dependent bathymetry and geoacoustic parameters. Bathymetry was taken from NOAA fathometer measurements, and describes a bottom with main channel depth from 6-17m depth (Figure 3.4).

Attenuation, sound speed, and density parameters were derived based on the results of boring studies [23] and laboratory analysis [24] carried out by the CRC as part of the survey work in preparation for construction.

Sound speed profiles were obtained from the results of boring geophysical measurements, such as in Figure 3.5, and were used directly in the model. Sediment 

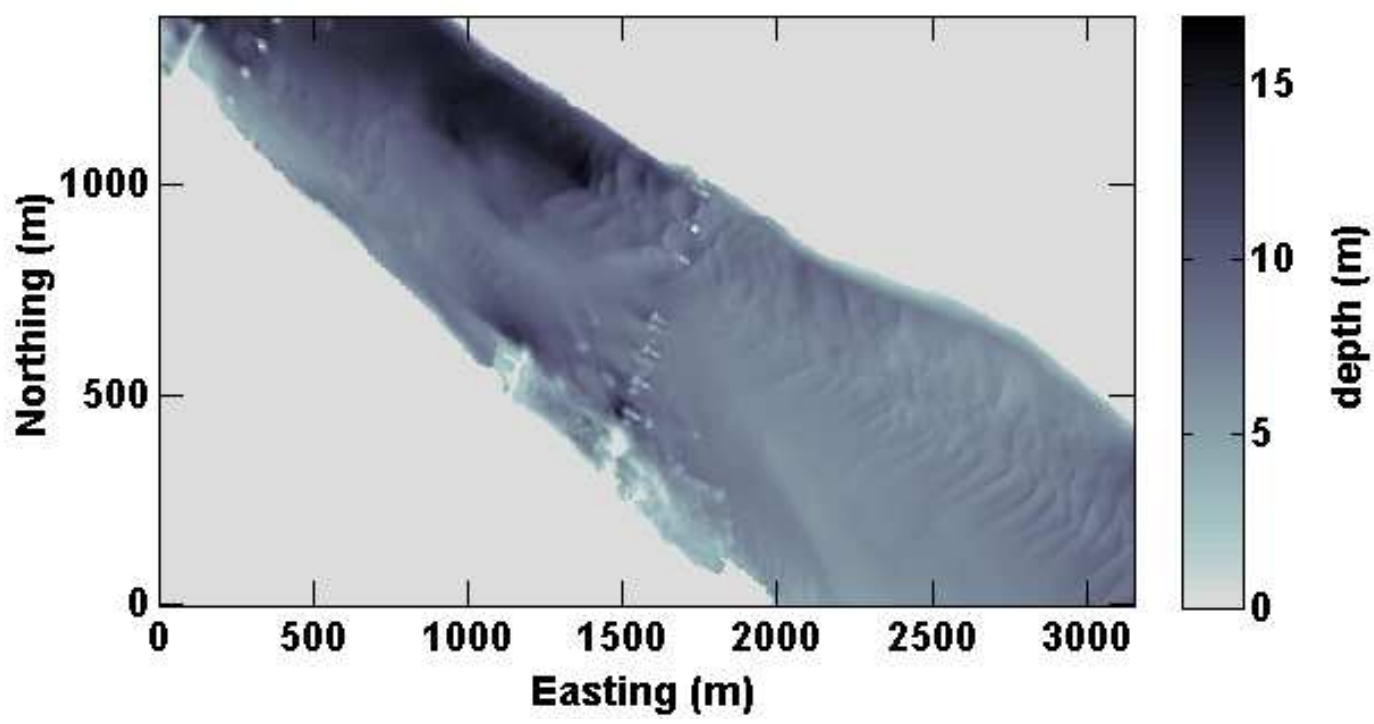

Figure 3.4: Columbia River Bathymetry obtained from NOAA fathometer measurements. Bathymetry transects are taken directly from this data and used in the model.

density was obtained by laboratory measurements that measured the water content, and therefore the porosity of the sediment. This was used in conjunction with the bulk density of each sediment layer described to give the density,

$$
\rho_{s}=\epsilon \rho_{w}+(1-\epsilon) \rho_{s b}
$$

where $\epsilon$ is the sediment porosity, $\rho_{s}$ is the sediment density, and $\rho_{s b}$ is the bulk sediment density. Sediment attenuation is based on the viscoelastic model of Hamiliton [26]. This model describes sediment attenuation that varies linearly with frequency,

$$
\alpha=\frac{k_{p} f}{c_{p}}
$$




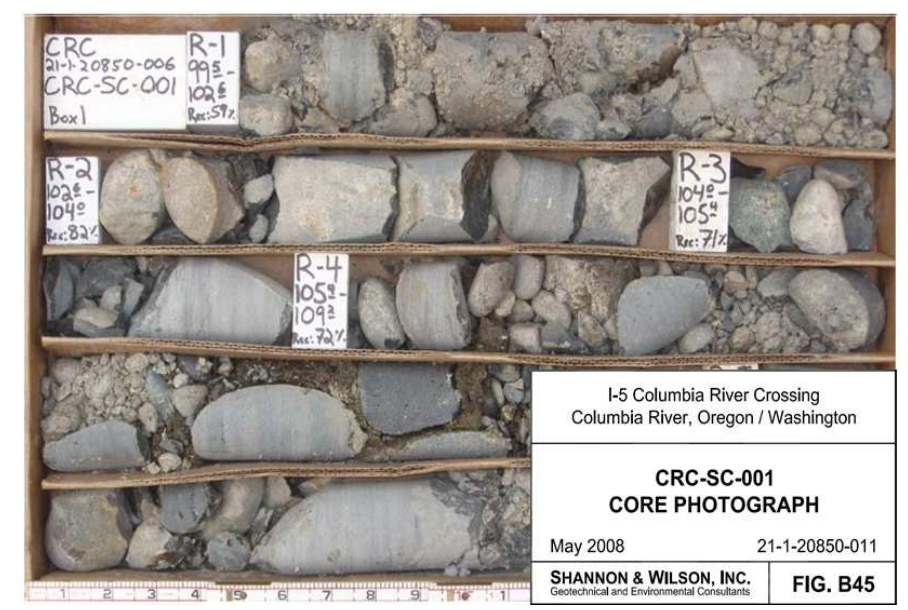

Figure 3.5: Example coring sample taken of the Troutdale Formation under the Columbia River.

where $\alpha$ is the attenuation in $\frac{d B}{\lambda}$ and $k_{p}$ is the loss parameter. The loss parameters used were taken from a table in the APL-UW High Frequency Ocean Environment Acoustic Models Handbook [27]. Values were chosen by matching the sediment description in the table as well as the ratio of density and velocity from the boring studies and equation (3.1), to a loss parameter.

For each pile, identical geoacoustic density and attenuation parameters were used, summarized in Table 3.1, although the depths of these sediment layers varied depending on the pile. The sound speed profiles used are shown in Figure 3.6, where the stark difference in the depths of the sediment layers can be clearly seen. These values were used directly in the model. For depths where sound speed values were not defined, the average value from the corresponding region was used.

The empirical source model requires that spectral weight functions, attenuation 
Table 3.1: Summary of Geoacoustic Parameters

\begin{tabular}{|l|l|l|}
\hline & Desity $(\mathrm{g} / \mathrm{cc})$ & Attenuation $(\mathrm{dB} / \lambda)$ \\
\hline Sand & 1.84 & 0.88 \\
\hline Gravel & 2.151 & $0.88-0.75$ (Interpolated) \\
\hline Troutdale Formation & 2.5 & 0.75 \\
\hline
\end{tabular}

parameters, and energy matching offsets be defined for each pile. The pile specifications are summarized in Table 3.2. The maximum frequency of the simulations was chosen so that the results of equation (2.19) was at least 0.97 , which resulted in the simulated bandwidth containing at least $97 \%$ of the waveform energy. A minimum frequency of $50 \mathrm{~Hz}$ was used for all simulations. For the larger diameter piles, the energy was more concentrated in the lower frequencies, as can be seen in the spectral weight functions in Figure 3.7, and this explains the lower maximum frequencies required to model larger piles. The larger piles also produced higher sound levels, which are reflected by the higher offset parameters. 


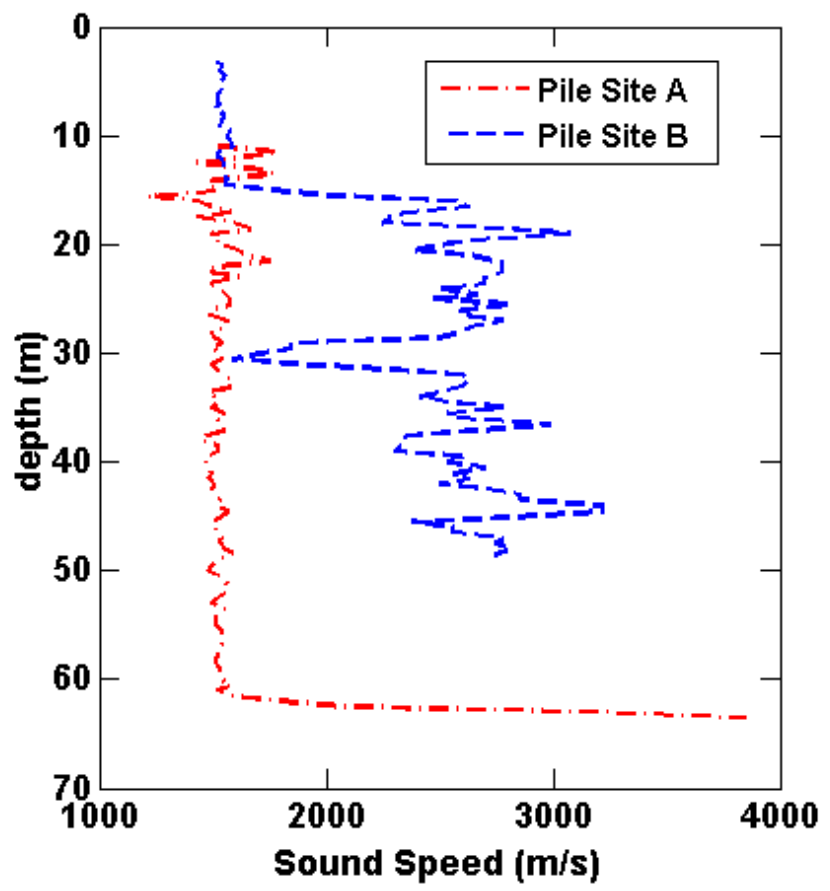

Figure 3.6: Sound speed profiles for sites A and B. The lower sound speed regions at shallow depth correspond to the sand layer, the rapid transition corresponds to the narrow gravel layer, and the high sound speed region corresponds to the Troutdale Formation.

Table 3.2: Pile Model Parameters

\begin{tabular}{|l|l|l|l|l|}
\hline Pile Name & B1 & B2 & A1 & A3 \\
\hline Diameter $(\mathrm{m})$ & 24 & 48 & 24 & 48 \\
\hline Length $(\mathrm{m})$ & 27.75 & 29.25 & 24.75 & 40 \\
\hline Wetted Length $(\mathrm{m})$ & 22.5 & 23.5 & 18.25 & 33.5 \\
\hline Gravel Depth $(\mathrm{m})$ & 14.5 & 14.5 & 60 & 60 \\
\hline Bedrock Depth $(\mathrm{m})$ & 15.5 & 15.5 & 62 & 62 \\
\hline Offset $(\mathrm{dB})$ & 90.2 & 96.7 & 91.1 & 100.0 \\
\hline Attenuation Factor & $1 / 3$ & $1 / 5$ & $2 / 5$ & $2 / 5$ \\
\hline Maximum Frequency $(\mathrm{Hz})$ & 2600 & 2050 & 2600 & 2100 \\
\hline
\end{tabular}


Pile B1

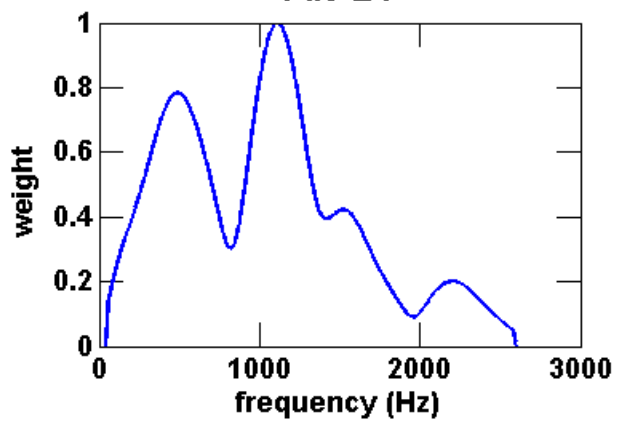

Pile A1

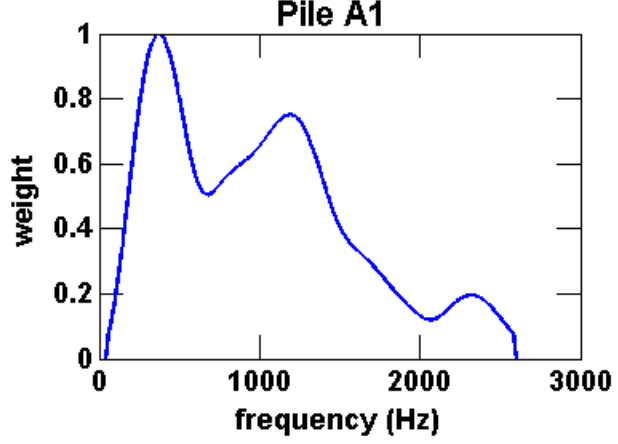

Pile B2

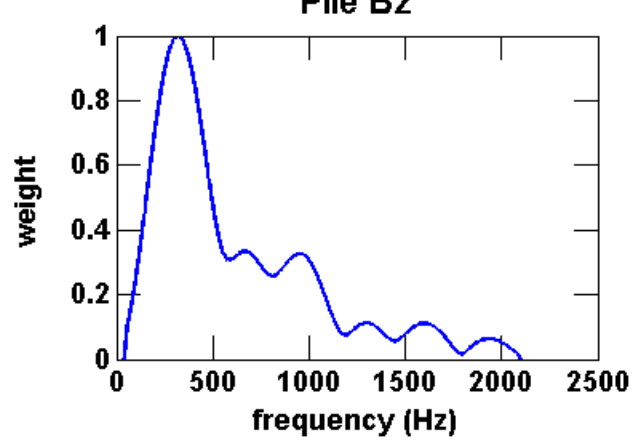

Pile A3

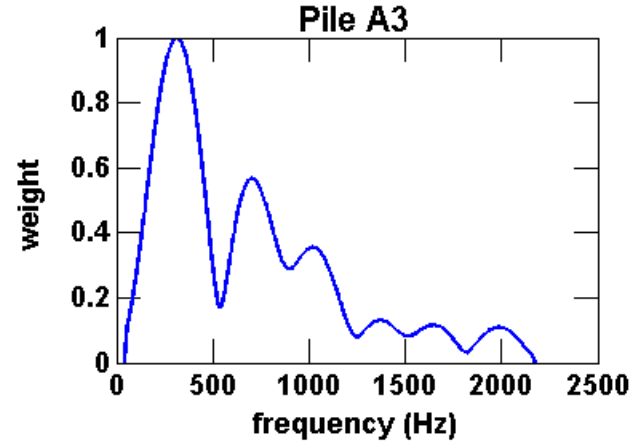

Figure 3.7: Spectral weight functions used for modeling. Curves are derived from waveforms observed $10 \mathrm{~m}$ for each pile.

\subsection{Experimental Data}

The acoustic data provided by David Evans and Associates [25] was contained in custom .wav files, each of which corresponded to a specific pile driving event and observation location. (i.e. pile B-2, 10m range). The sampling frequency of the data was dependent on the observation location (either $44.1 \mathrm{kHz}$ or $96 \mathrm{kHz}$ ), and the sensitivity of the hydrophones was mostly flat across the spectral regions of interest.

After discarding an 80 bit header file, the data was read into the MATLAB workspace as 24 bit data. To convert from arbitrary data units to $\mathrm{Pa}$, a calibration 
factor was applied based on the sensitivity of the hydrophone. Also, the last 500 bits of data contained additional file header information that was discarded.

Pile Driving Data Record

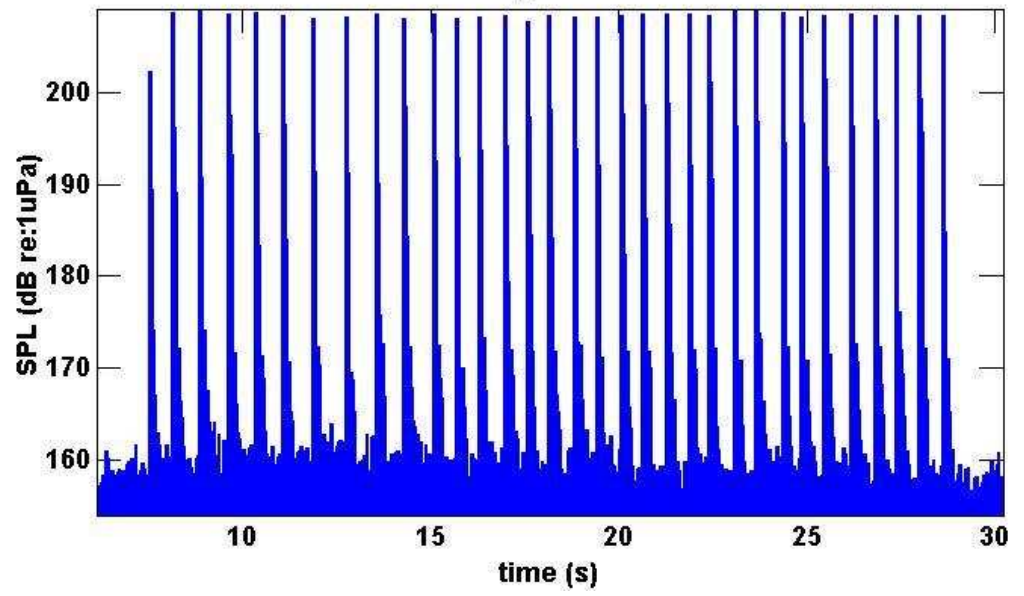

Figure 3.8: Example pile driving data record, which shows the SPL of several consecutive pile driving impacts.

Each .wav file contained several consecutive blows, as in Figure 3.8. Individual impacts were isolated using a threshold method, and the average of the individual waveforms was used for analysis. In this method, a percentage of the maximum value in the data file was specified, and all indices that corresponded to these values were isolated. The first index in a group of adjacent such indices gave the start of the waveform. (the actual waveform was taken as a set number of points before this first index.) The length of the waveform was chosen to be $0.1 \mathrm{~s}$, which typically corresponded to $99 \%$ of the total waveform energy.

Individual waveforms were used to generate an ensemble average waveform, SEL, and PSD, shown in Figure 3.9. The PSD was calculated by the Welch method, 
(A)

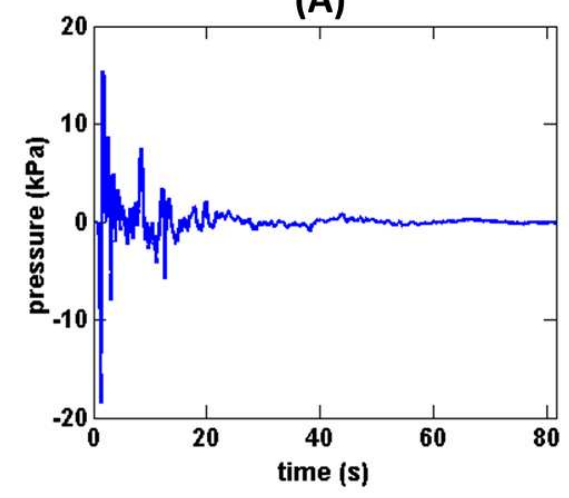

(B)

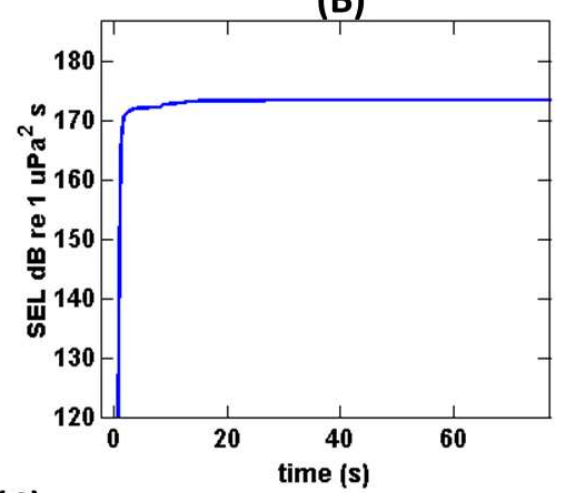

(C)

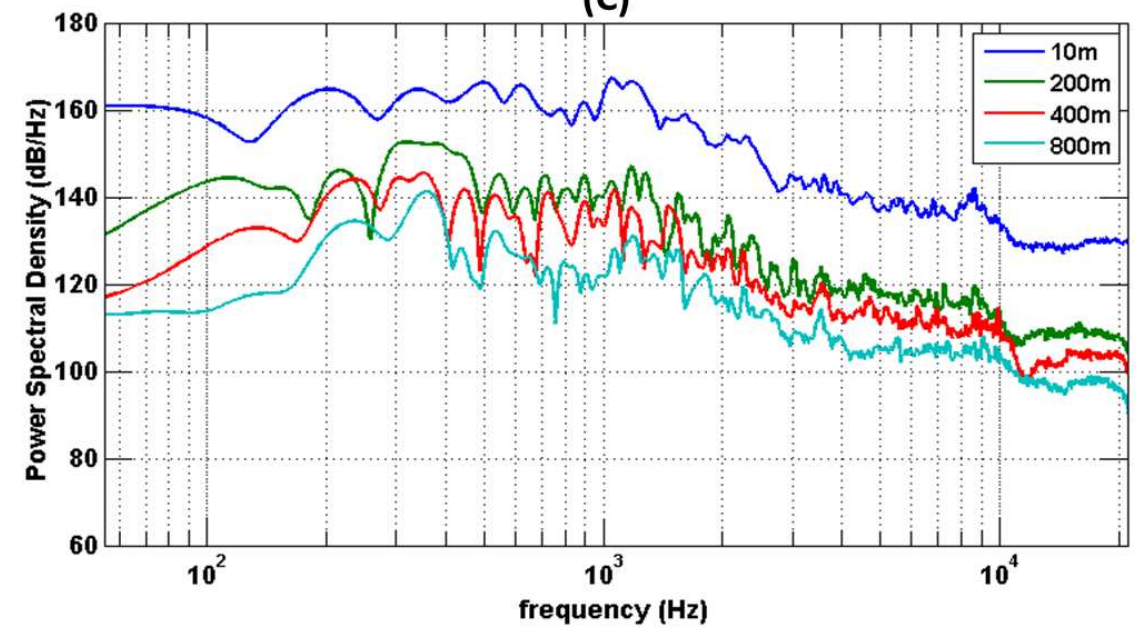

Figure 3.9: Example ensemble averaged time domain waveform (A), sound exposure level (B), and PSD (C), for pile B1. The waveform and SEL are from the $10 \mathrm{~m}$ observation. PSD curves correspond to the four upstream measurement locations.

using 2048 point segments and a Hamming window with the corresponding window correction factor applied. This specific analysis was chosen to match the analysis done by the David Evans and Associates engineers. The ensemble averaged time domain waveform was used to calculate SPL and SEL. 


\section{Chapter 4}

\section{Comparison to Observed Data}

In this chapter the validity of the source models and propagation model are evaluated by comparisons with measured data in a variety of environmental conditions. The simulated results correspond to two-dimensional (range, depth) simulations calculated along the path of the observation locations for both pile driving sites $\mathrm{A}$ and B, shown in Figure 3.2. For each observation site, simulations were computed for comparison to the data from the 24 and 48 inch diameter piles at 5 locations each. This results in 20 data points for comparison. Simulation parameters were set to match the environmental conditions detailed in Chapter 3.

For each simulation, convergence was tested (Appendix B) at the minimum, middle, and maximum frequencies. Also, each frequency solution was visually inspected for glitches. In all modeling a depth step of $0.25 \mathrm{~m}$ and range step of $0.5 \mathrm{~m}$ was used, which was a uniform, convergent grid at all frequencies.

\subsection{Source Model Comparisons}

For both the empirical and FDTD source models, the source functions were visualized using a short-range synthesis of the broadband RAM-PE solutions. In Figures 4.1 and 4.2, the transmission loss is shown over a two-dimensional area, at time steps of 5, 10, 20 and 30ms. In addition to being a detailed illustration 
of the source functions, these plots verified that the intended source function was properly implemented. Proper implementation was verified by comparing the theoretical predictions in Chapter 2 to the simulated results, and also by verifying that the simulation was free of artifacts caused by improper implementation or lack of convergence.

In the visualization of the empirical source model in Figure 4.1, conical waves can be seen to radiate uniformly into the environment at the angles predicted by equation (2.20). This is contrasted by the visualization of the FDTD source model shown in Figure 4.2. For the first approximately 10ms, very little energy is radiated into the environment. This corresponds to the period of time when the hammer is in contact with the pile, but the bulk of the force has not yet been applied. When the main force is applied, a conical wave is radiated at a similar incidence angle to the first arrival of the empirical model. However, after the first arrival, the modal response of the pile dominates, and the conical wave structure of the empirical model is no longer present. 

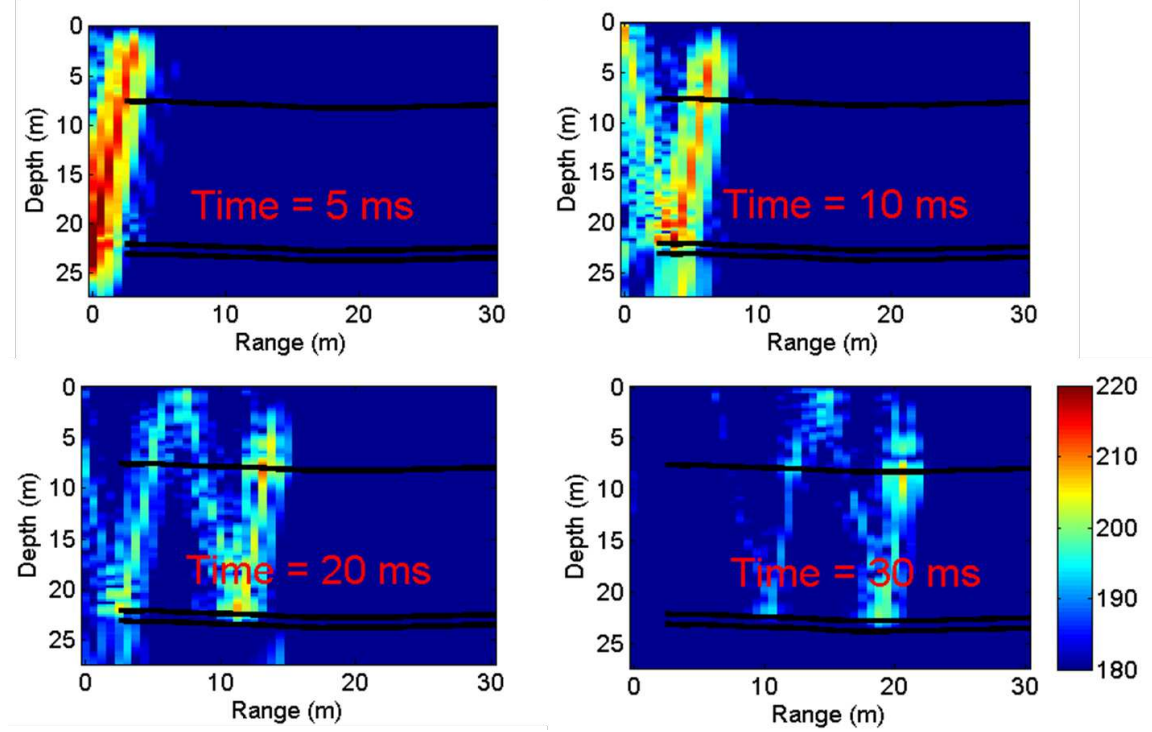

Figure 4.1: Short-range propagation still frames using the empirical source model to represent pile B1. The angled waves were emitted from the pile for a time period that included 4 arrivals. The black lines demarcate the sediment layer boundaries. The top layer is the water column, followed by sand, gravel and the Troutdale Formation, descending downward.
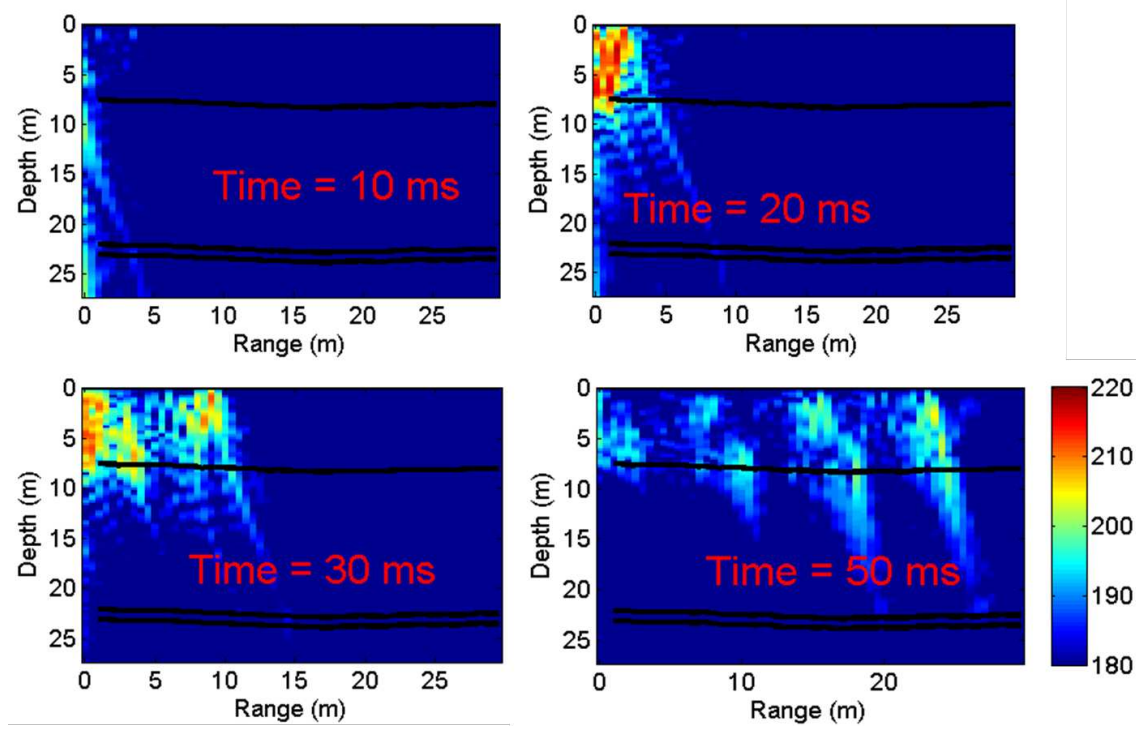

Figure 4.2: Short-range propagation still frames using the FDTD source model to represent pile B1. The lines demarcate the sediment layer boundaries. The top layer is the water column, followed by sand, gravel and the Troutdale Formation, descending downward. 
Probably the most important distinction between the source models is the amount of energy radiated into the sediment. While the empirical source model radiates energy uniformly into the water column and sediment, the FDTD model radiates very little into the sediment. This is a topic that needs to be addressed in future research, and is of particular consequence to methods of attenuating the underwater sound. Typical methods of sound attenuation reduce only sound that has been emitted in the water column. The portion of sound produced in the water column as compared to in the sediment could have significant implications for the viability of such attenuation methods.

\subsection{PSD and SPL Comparison}

Power Spectral Density (PSD) is compared to acoustic data in Figures 4.3-4.6, where each figure corresponds to a different pile. Comparisons show good agreement with both the FDTD and Empirical source models. While the empirical source model forces good agreement at the close range observation with the spectral weighting function, the FDTD source model by contrast has no such weighting. Beyond the close range comparisons, the continued favorable agreement in roll off show that the geoacoustic model is attenuating the waveforms accurately across the whole frequency band.

While some of the deviations between the model and acoustic data are caused by environmental simplifications inherent in the model, the main cause of disagreement 
is due to peaks and nulls. Peaks and nulls are produced by areas of constructive and destructive interference caused by multipath interactions, and occur at integer multiples of a wavelength. In the band where discrepancies primarily occur, between 500 to $2600 \mathrm{~Hz}$, the wavelength varies from 3 to $0.57 \mathrm{~m}$, which is well within the uncertainty in the locations of the acoustic observations and bathymetry measurements. 
(A)

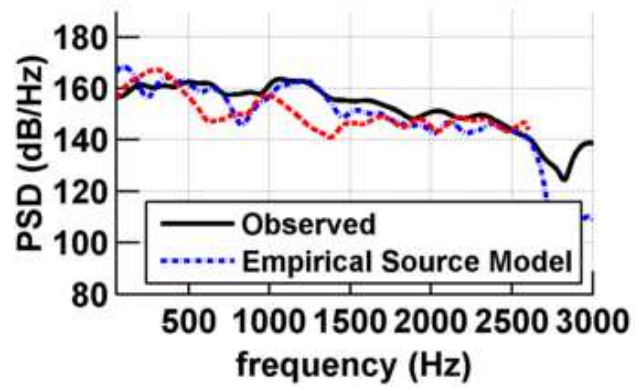

(C)

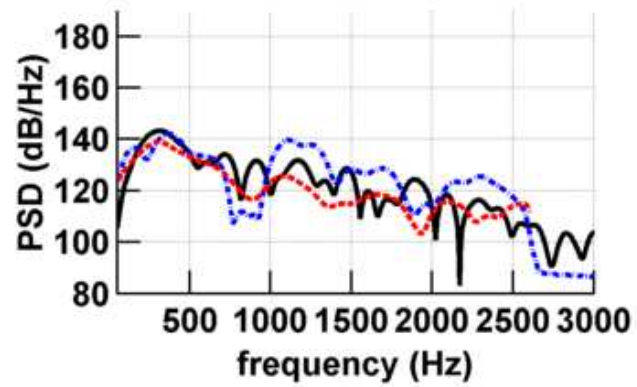

(E)

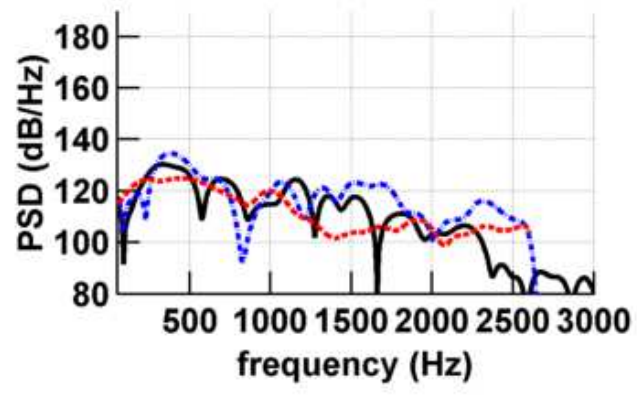

(B)

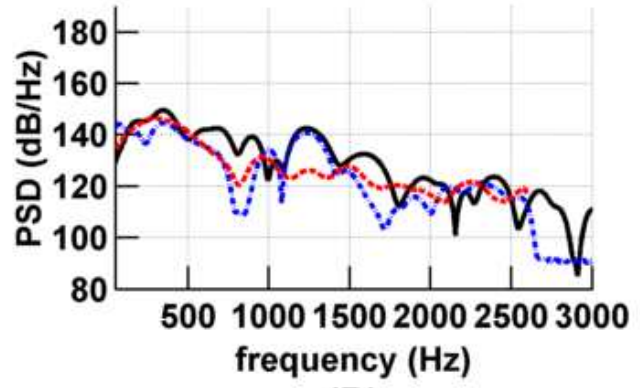

(D)

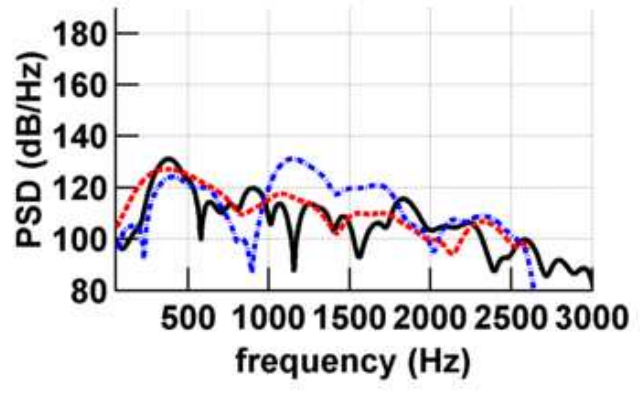

Figure 4.3: PSD comparisons for pile B1. Panel A corresponds to the $10 \mathrm{~m}$ Observation site, B to $200 \mathrm{~m}$, C to $400 \mathrm{~m}$, D to $800 \mathrm{~m}$, and $\mathrm{E}$ to the $800 \mathrm{~m}$ site in the opposite direction. 

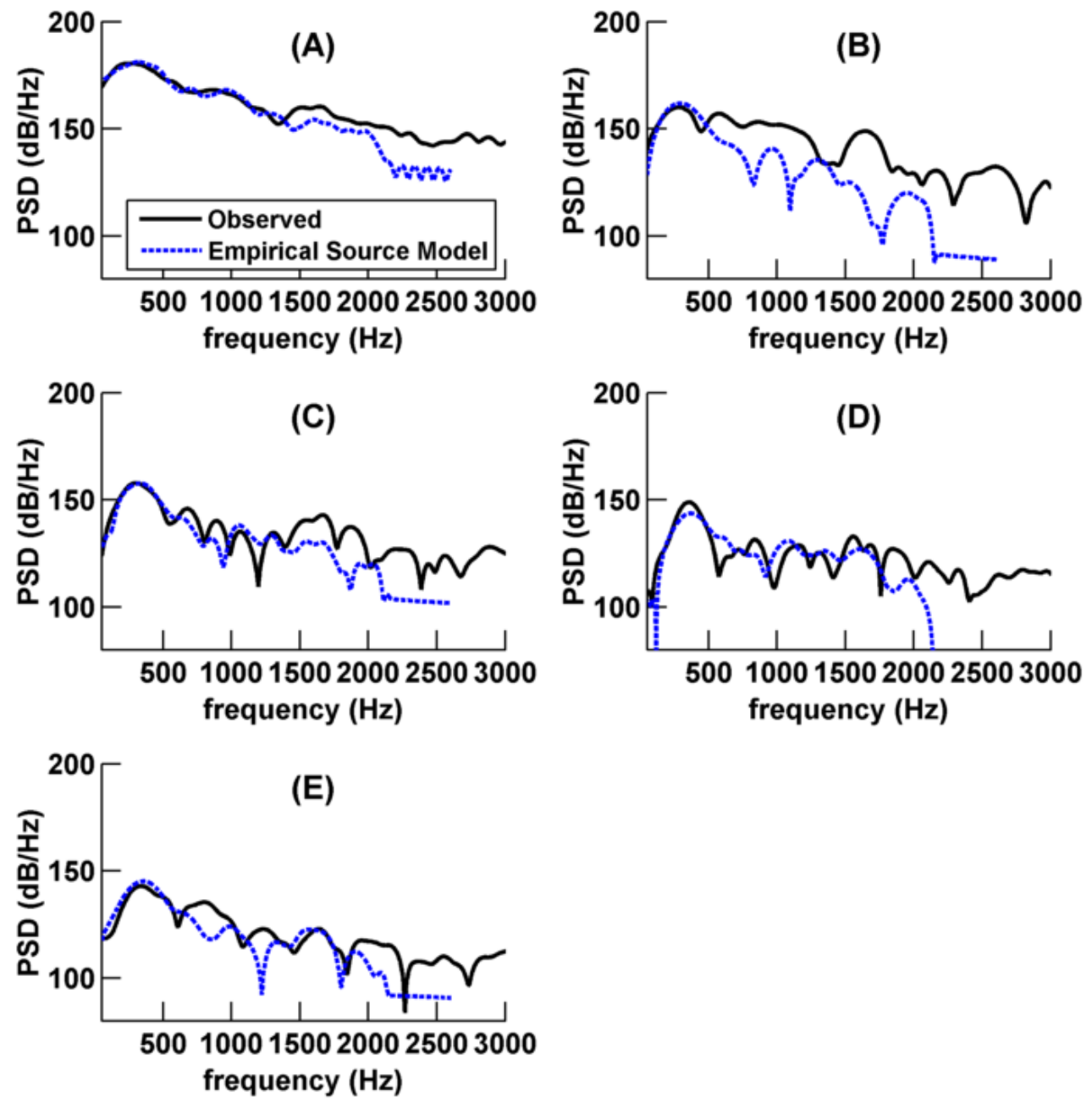

Figure 4.4: PSD comparisons for pile B2. Panel A corresponds to the $10 \mathrm{~m}$ Observation site, B to $200 \mathrm{~m}, \mathrm{C}$ to $400 \mathrm{~m}, \mathrm{D}$ to $800 \mathrm{~m}$, and $\mathrm{E}$ to the $800 \mathrm{~m}$ site in the opposite direction. 

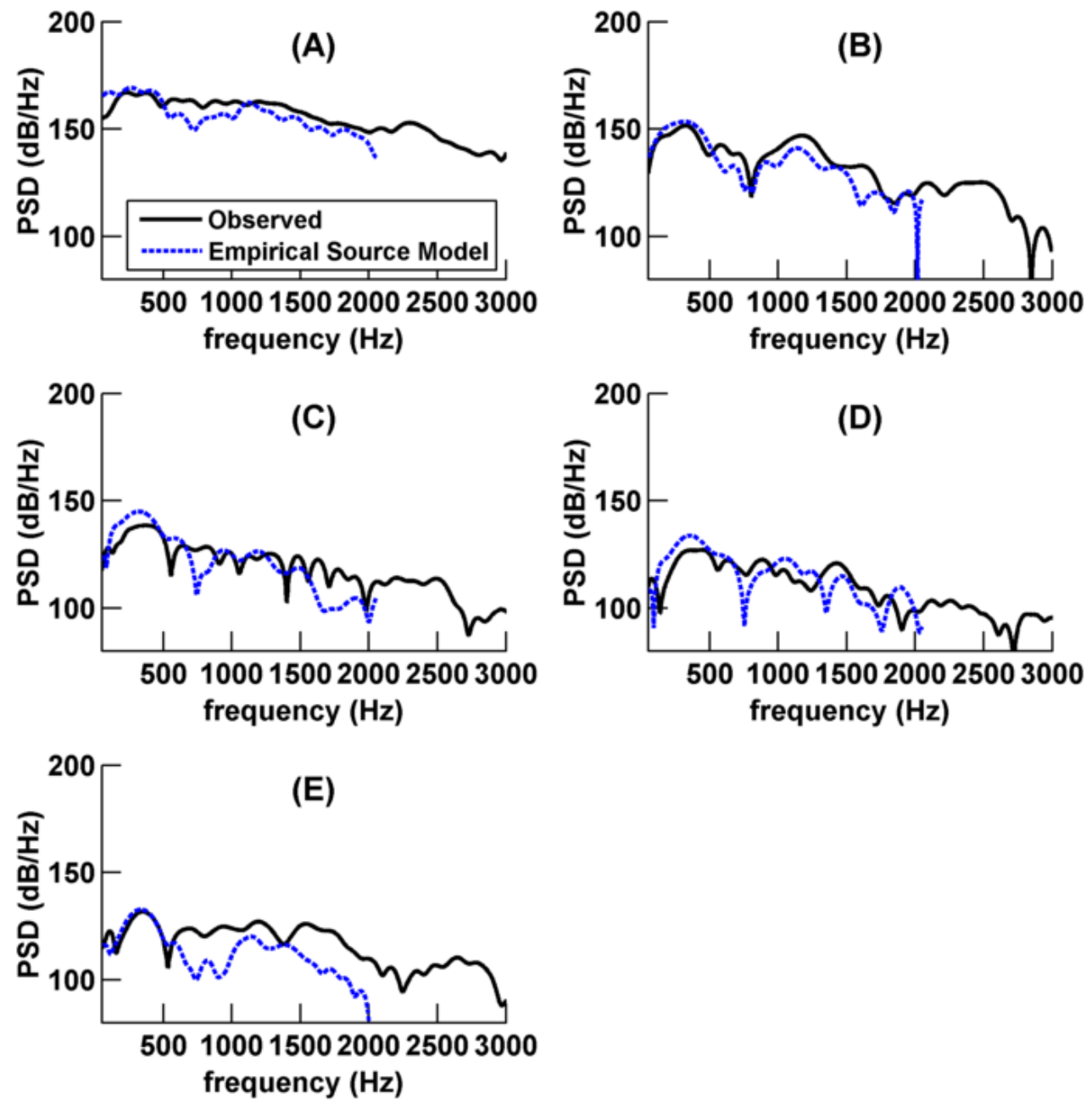

Figure 4.5: PSD comparisons for pile A1. Panel A corresponds to the $10 \mathrm{~m}$ Observation site, B to $200 \mathrm{~m}, \mathrm{C}$ to $400 \mathrm{~m}, \mathrm{D}$ to $800 \mathrm{~m}$, and $\mathrm{E}$ to the $800 \mathrm{~m}$ site in the opposite direction. 

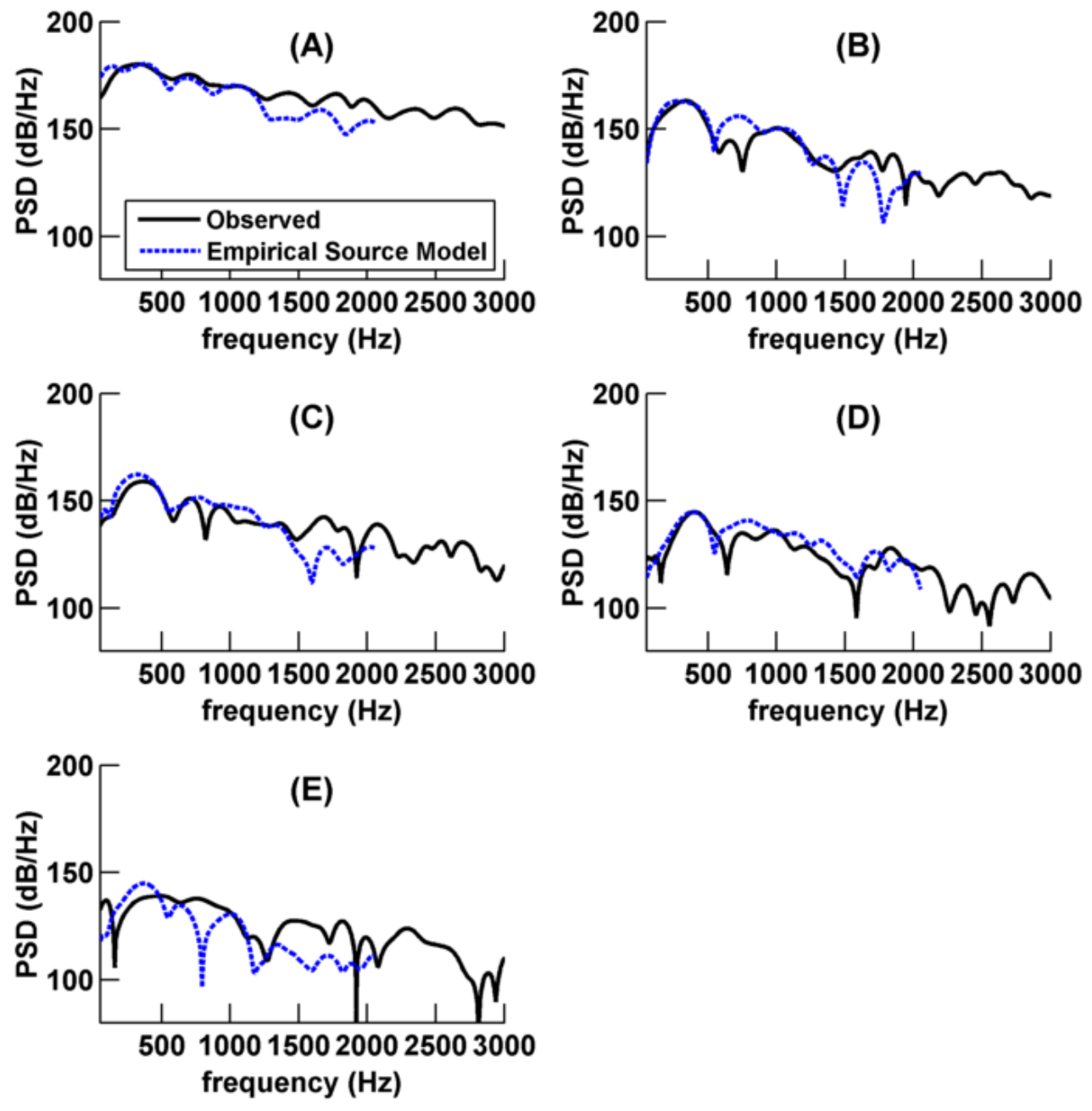

Figure 4.6: PSD comparisons for pile A3. Panel A corresponds to the 10m Observation site, B to $200 \mathrm{~m}$, C to $400 \mathrm{~m}$, D to $800 \mathrm{~m}$, and $\mathrm{E}$ to the $800 \mathrm{~m}$ site in the opposite direction.

SPL comparisons also showed good agreement in the absolute levels, shown in Figures 4.7-4.10. Matching specific peaks was unreliable beyond very close range, due to uncertainty in measurement locations. The empirical source model showed 
the best agreement in matching the peaks, because the attenuation factor could be chosen to best match the close range waveform.
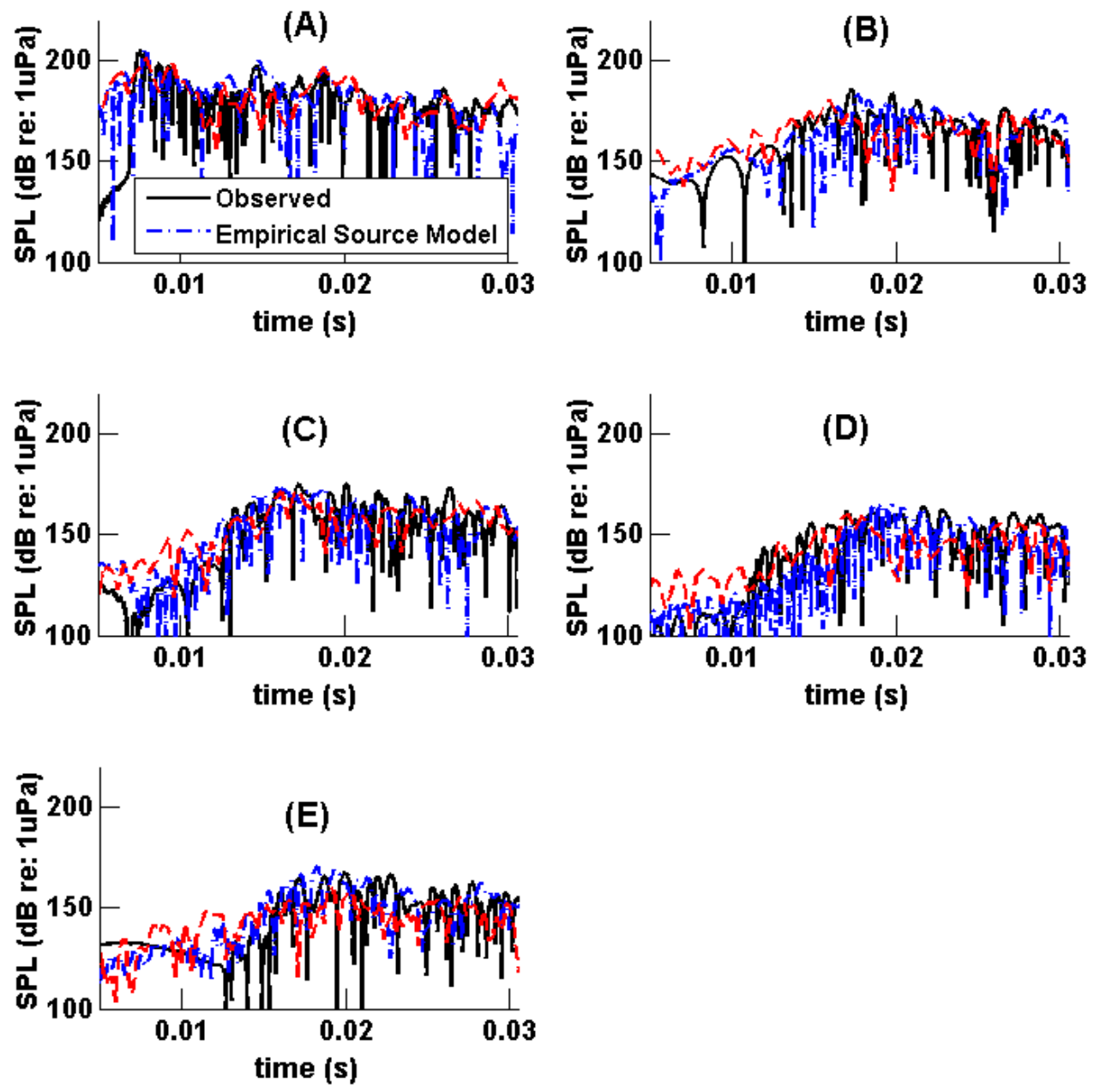

Figure 4.7: SPL comparisons for pile B1. Panel A corresponds to the 10m Observation site, B to $200 \mathrm{~m}, \mathrm{C}$ to $400 \mathrm{~m}$, D to $800 \mathrm{~m}$, and $\mathrm{E}$ to the $800 \mathrm{~m}$ site in the opposite direction. 

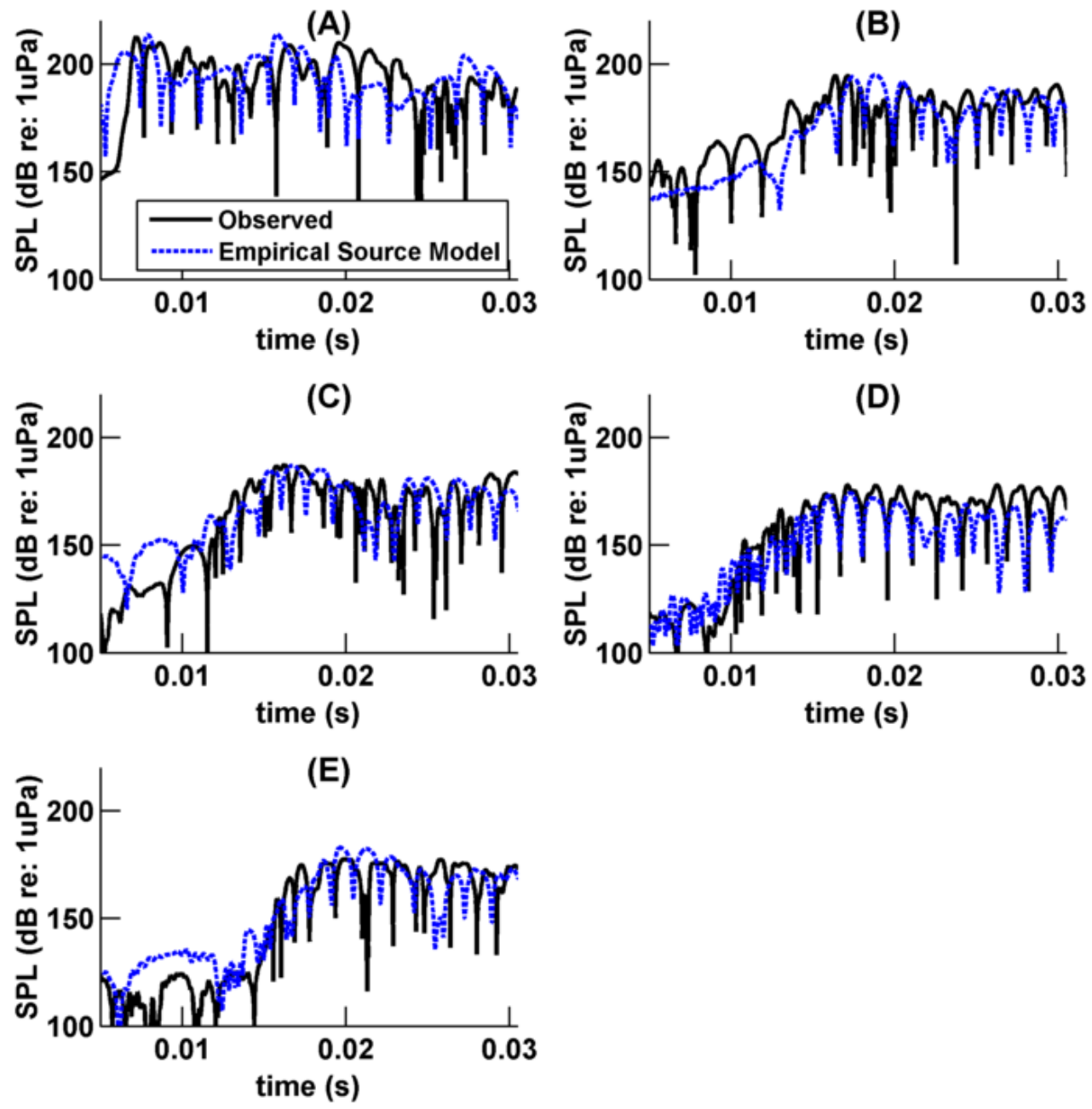

Figure 4.8: SPL comparisons for pile B2. Panel A corresponds to the 10m Observation site, B to $200 \mathrm{~m}, \mathrm{C}$ to $400 \mathrm{~m}, \mathrm{D}$ to $800 \mathrm{~m}$, and $\mathrm{E}$ to the $800 \mathrm{~m}$ site in the opposite direction. 

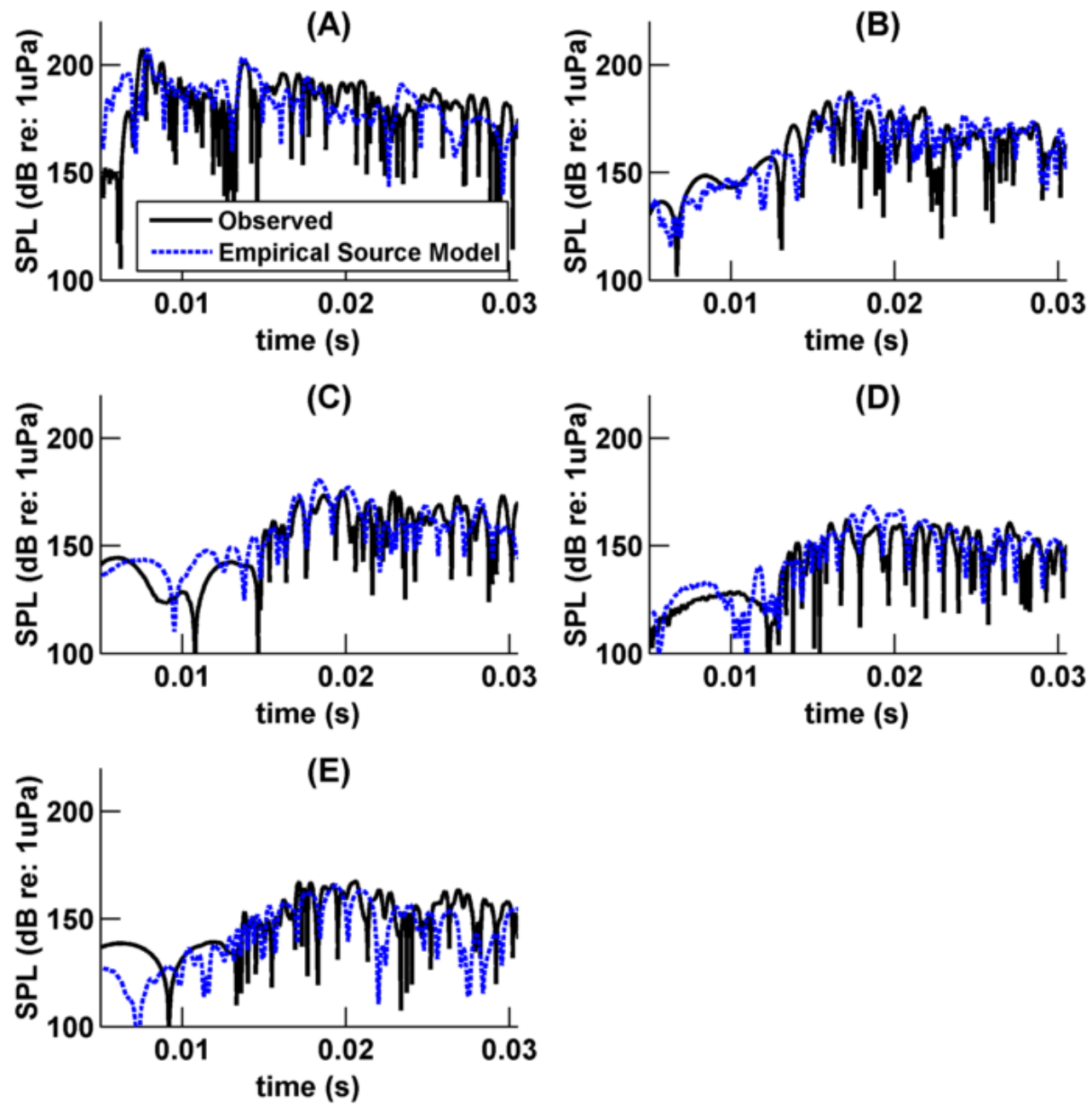

Figure 4.9: SPL comparisons for pile A1. Panel A corresponds to the $10 \mathrm{~m} \mathrm{Ob-}$ servation site, B to $200 \mathrm{~m}, \mathrm{C}$ to $400 \mathrm{~m}, \mathrm{D}$ to $800 \mathrm{~m}$, and $\mathrm{E}$ to the $800 \mathrm{~m}$ site in the opposite direction. 

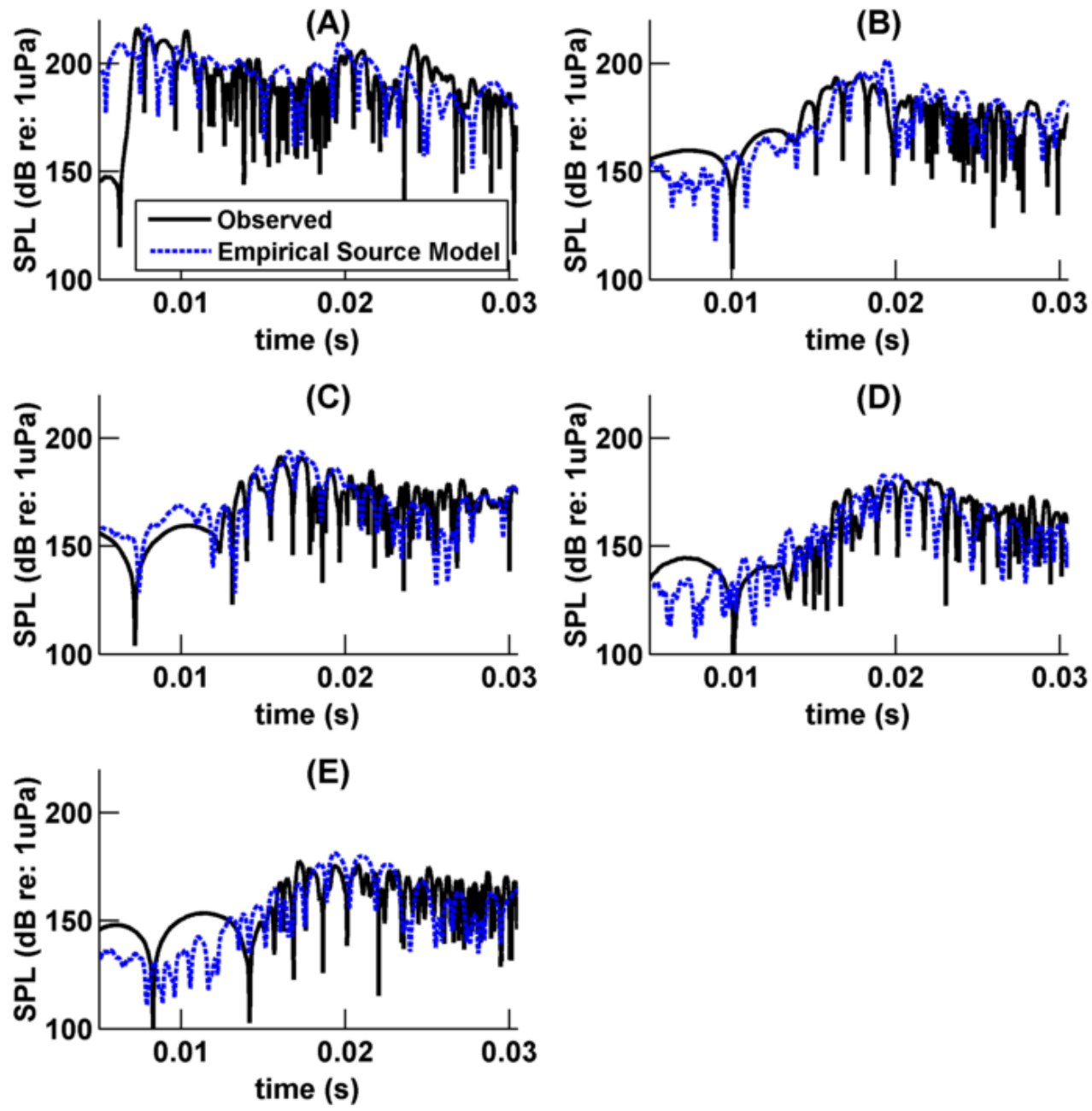

Figure 4.10: SPL comparisons for pile A3. Panel A corresponds to the 10m Observation site, B to $200 \mathrm{~m}$, C to $400 \mathrm{~m}$, D to $800 \mathrm{~m}$, and $\mathrm{E}$ to the $800 \mathrm{~m}$ site in the opposite direction.

\subsection{Sound Exposure Level Comparisons}

The SEL predicted by the model is compared to observations graphically in Figure 4.11 and numerically in Table 4.1. The agreement is very encouraging. In 22 of 25 
comparisons, the model agrees with observation to within $4 \mathrm{~dB}$, where all instances of disagreement greater than $4 \mathrm{~dB}$ occurred at the $800 \mathrm{~m}$ observation locations. Also, all instances of large disagreement occurred at pile site $\mathrm{B}$, where there is more variance and uncertainty in the sediment. At the closer observation locations, the agreement was much better, within $3 \mathrm{~dB}$ at all observation locations. This suggests a high level of reliability in predictions within 400m.

Table 4.1: Sound Exposure Level Summary

\begin{tabular}{|c|c|c|c|c|c|c|}
\hline $\begin{array}{l}\text { Observation } \\
\text { Distnace }\end{array}$ & $10 \mathrm{~m}$ & $200 \mathrm{~m}$ & $400 \mathrm{~m}$ & $800 \mathrm{~m}$ & $-800 m$ & $\begin{array}{c}\text { Average } \\
\text { Pile Error }\end{array}$ \\
\hline B1 Empirical & 173.33 & 155.29 & 147.85 & 136.84 & 140.71 & \\
\hline B1 FDTD & 174.76 & 153.31 & 146.30 & 134.51 & 134.08 & \\
\hline B1 Observed & 173.50 & 155.74 & 149.89 & 139.36 & 142.12 & \\
\hline Empirical Difference & 0.17 & 0.44 & 1.34 & 2.52 & 1.41 & 1.18 \\
\hline FDTD Difference & 1.26 & 2.44 & 2.89 & 4.84 & 8.04 & 3.90 \\
\hline$\overline{\text { B2 Empirical }}$ & 185.97 & 168.43 & 161.27 & 148.17 & 156.60 & \\
\hline B2 Observed & 185.99 & 168.25 & 162.58 & 156.21 & 155.85 & \\
\hline Empirical Difference & 0.02 & 0.18 & 1.31 & 8.04 & 0.76 & 1.67 \\
\hline A1 Empirical & 175.84 & 158.66 & 151.49 & 140.76 & 139.25 & \\
\hline A1 Observed & 175.87 & 156.53 & 149.32 & 137.27 & 142.09 & \\
\hline Empirical Difference & 0.03 & 2.13 & 2.17 & 3.49 & 2.84 & 0.96 \\
\hline$\overline{\text { A3 Empirical }}$ & $\overline{186.72}$ & 169.61 & 165.45 & 155.33 & 154.24 & \\
\hline A3 Observed & 186.80 & 166.53 & 162.90 & 154.87 & 153.27 & \\
\hline Empirical Difference & 0.08 & 2.72 & 2.55 & 0.47 & 0.97 & 1.32 \\
\hline $\begin{array}{l}\text { Distance Average } \\
\text { Error }\end{array}$ & 0.10 & 0.90 & 0.32 & 1.94 & 1.94 & \\
\hline
\end{tabular}

Summary of cumulative sound exposure levels for each pile and observation site, as well as the deviations between the model and observations. The differences show the $\mathrm{dB}$ discrepancy between the model and observations, and the average of the difference is shown in the Average Pile Error column. The average difference at each measurement distance is shown in the Distance Average Error row. The average of all discrepancies is $2.17 \mathrm{~dB}$. All entries are in units of $\mathrm{dB}$ re: $1 \mu \mathrm{Pa}^{2} s$. 

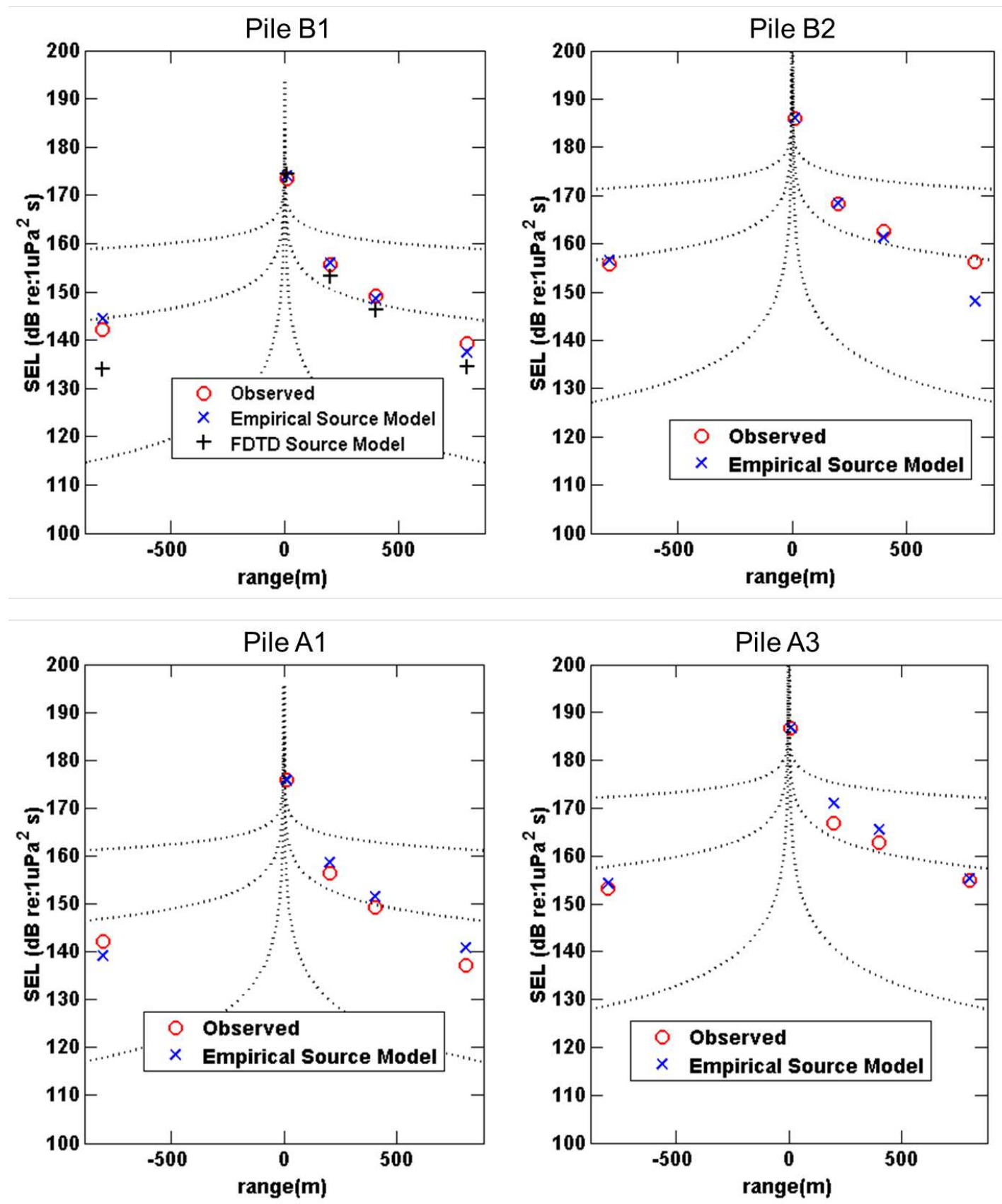

Figure 4.11: Sound exposure level summaries for each pile. The dotted lines correspond to results using the practical spreading model. The top line corresponds to an $\mathrm{F}$ factor of 5 and the bottom an $\mathrm{F}$ of 20 . The middle line is a fit to the data, and corresponds to an $\mathrm{F}$ of 10.5 . 


\section{Chapter 5}

\section{Applications}

The previous chapter established the validity of the source models and the propagation model by comparing results with measured data along multiple transects. Subsequently the model was extended to simulate the SEL in all directions surrounding the pile driving location, and to evaluate the effects of bathymetry and sediment layer thickness. Contour plots were generated from the solutions of several simulations. The effects of environmental conditions were quantified by comparing identical simulations, with only the parameter of interest varied. The simulations in this chapter demonstrate the relatively strong dependence of SEL on local environmental conditions and suggest that detailed environmental data is necessary to accurately predict areas where monitoring of pile driving activities is necessary.

\subsection{Contour Plots}

Contour plots were used to demonstrate the improvement upon the predictions produced by the practical spreading model. These plots show the results of several simulations, run about a common origin point, and predict SEL over large areas of the Columbia River. While the practical spreading model would simply produce concentric circles surrounding the pile driving site, significant variation from this 
can be seen in the contour plots. The sources of the irregularities are the inhomogeneities in the environment. That is, the variations in sediment depths and bathymetry, which are examined in greater detail in Sections 5.2 and 5.3.

Contour plots are shown for piles B1 (Figure 5.1) and A3 (Figure 5.2). In general, the variation is greater on the north riverside, where the bedrock layer is much shallower and bathymetry variation is greater. One interesting section is to the northeast of the pile in Figure 5.1. While deeper water causes less attenuation over long range, this area produces a very sudden attenuation. This particular discrepancy is caused by the waveform experiencing spreading greater than cylindrical spreading in this region, due to the suddenly deeper water. The effect of these variations underscores the need for more advanced predictions of this nature, since the distance required for sound levels to fall below a given threshold can vary greatly depending on the bearing angle, which is not accounted for in the practical spreading model. 


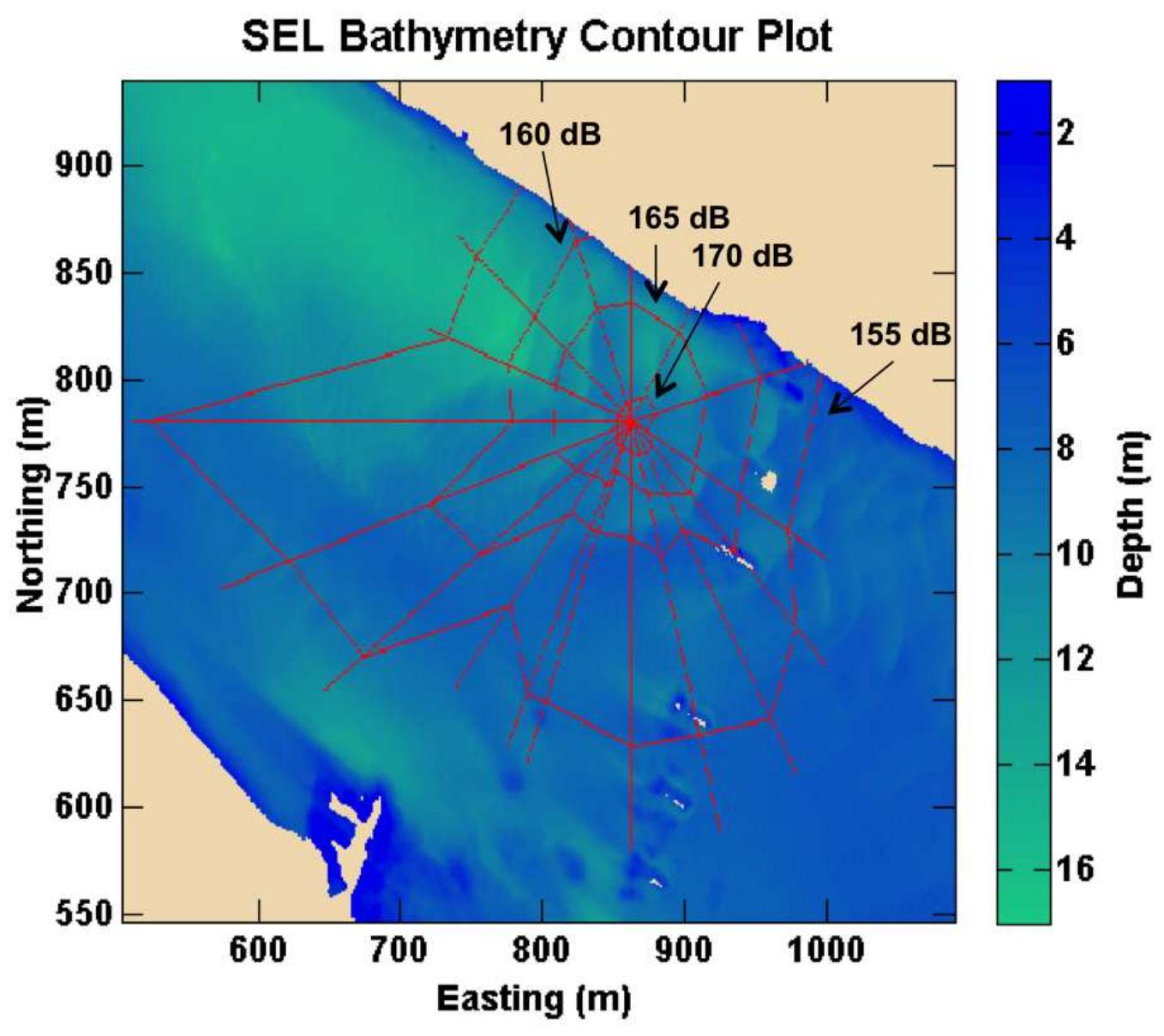

Figure 5.1: SEL contour plot about pile B1. The radial lines demarcate twodimensional simulation results, and the lines connecting radials connect points of equivalent SEL. 


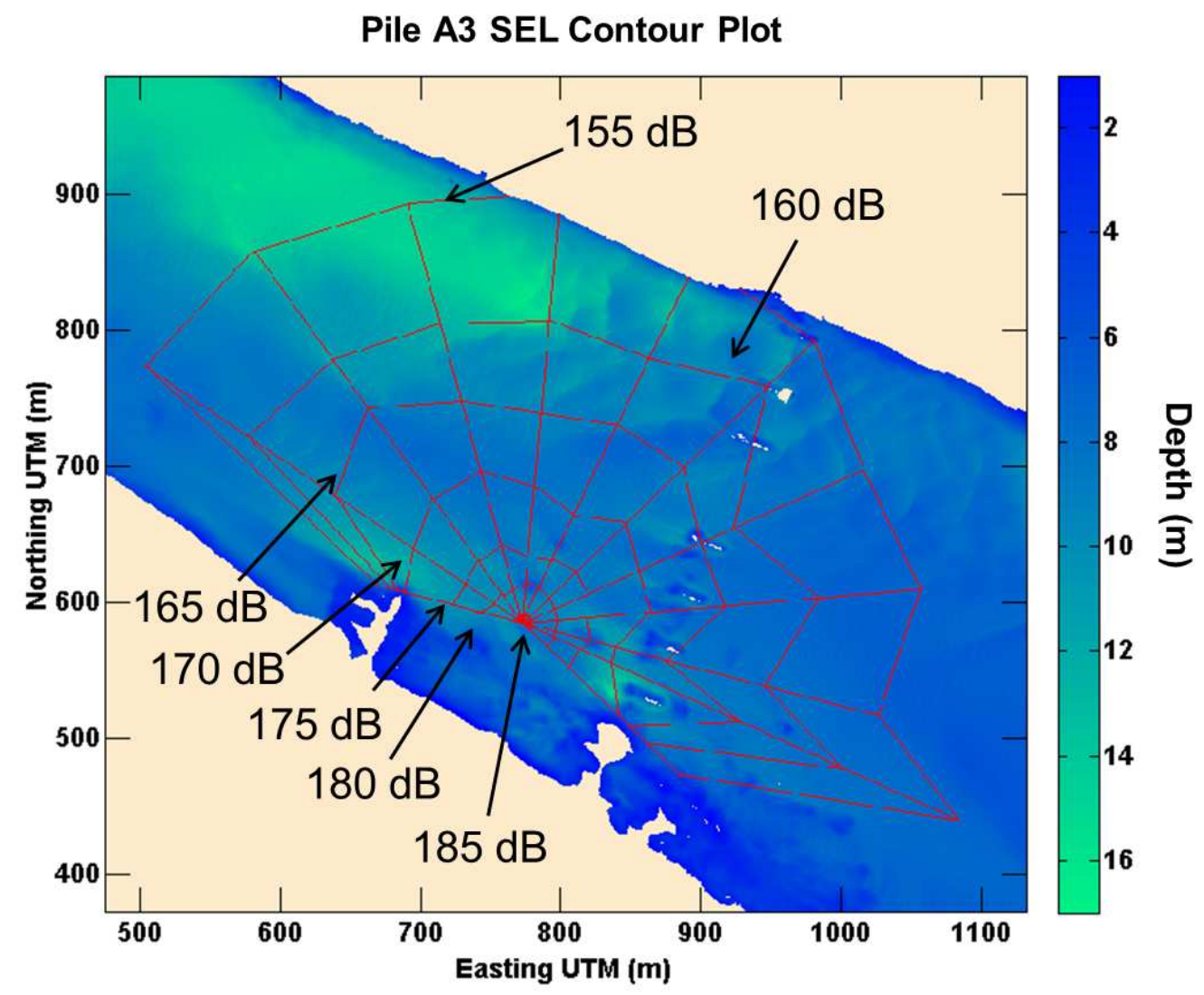

Figure 5.2: SEL contour plot about pile A3. The radial lines demarcate twodimensional simulation results, and the lines connecting radials connect points of equivalent SEL.

\subsection{Bathymetry Effect}

The effect of bathymetry on SEL was studied by selecting several characteristic test cases from the Columba River bathymetry. The test cases shown in Figure 5.3 were sections of bathymetry that encompassed the extremes of bathymetry variation in the neighborhood of pile driving operations. The effect of variations in bathymetry was quantified by calculating otherwise identical simulations, with the different 
pieces of bathymetry from the Columbia River used. Pieces of bathymetry were chosen to include as many of the features present in the environment as possible.
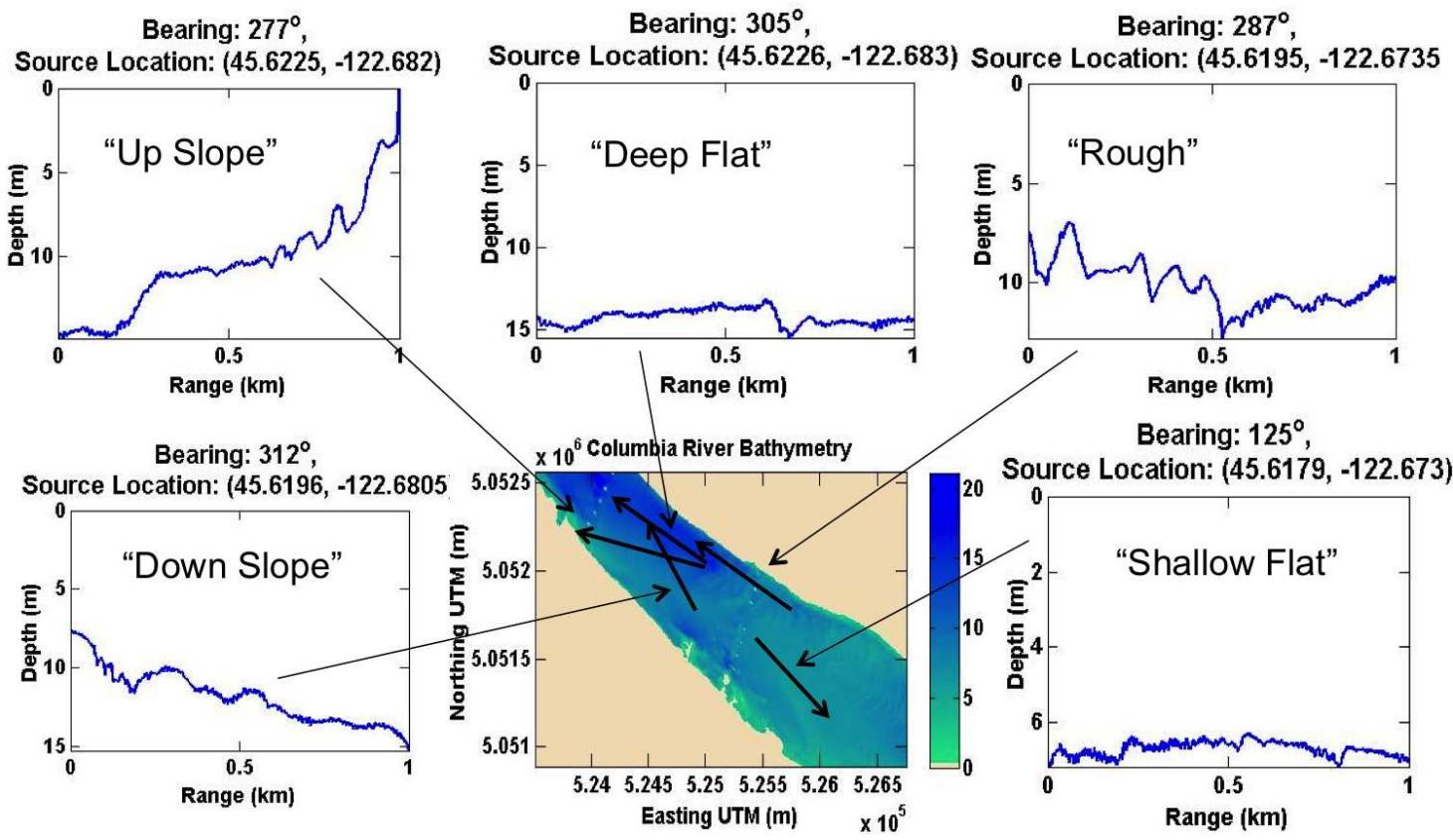

Figure 5.3: Five bathymetry test cases, located near the I-5 Columbia River crossing. Test cases encompass the deep and shallow extremes, as well as the extremes for roughness and sloping bottoms found in the neighborhood of the construction site.

The comparisons of the simulations are shown in Figures 5.4 and 5.5. While the bathymetry does effect SEL at localized ranges by as much as $5 \mathrm{~dB}$, the long range SEL shows little variation based on sloping terrain or roughness present in the Columbia River. The Bathymetry feature that does affect the SEL over long ranges appears to be only the absolute depth of the water column. This is caused by increased sediment-water reflections, over long ranges. 


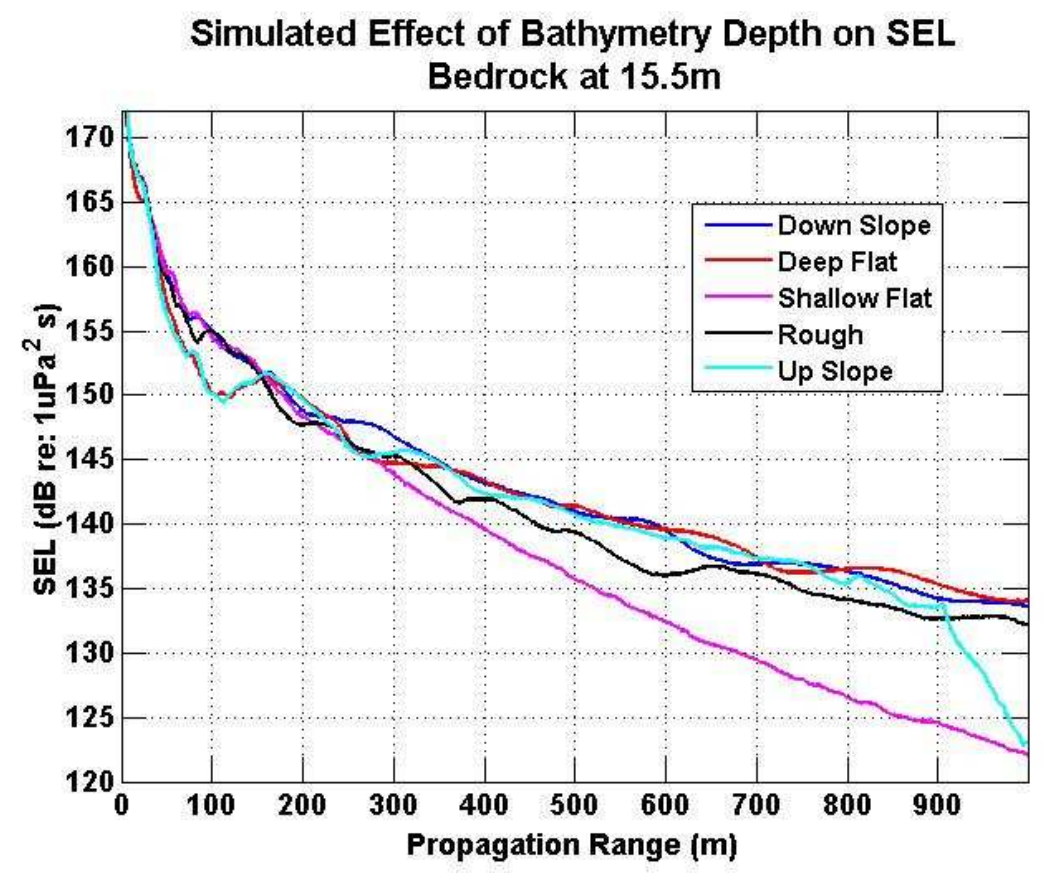

Figure 5.4: Range dependent SEL at a depth of 3.5m, for each bathymetry test case.

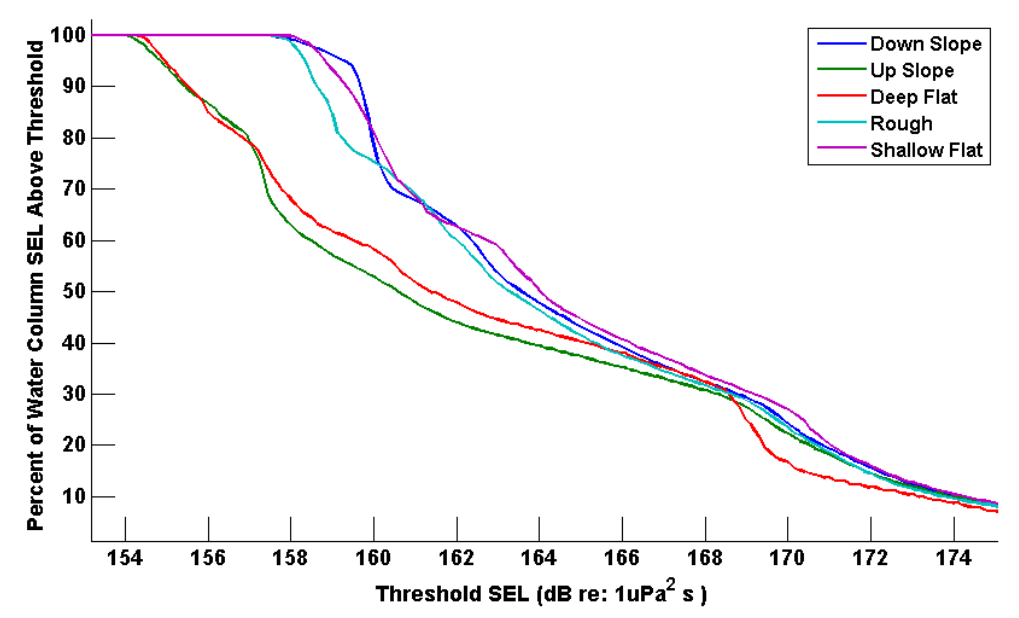

Figure 5.5: Statistical threshold plot comparing the percent of the water column SEL above certain thresholds, for the different test cases. The region of comparison comprises the water column from range $100 \mathrm{~m}$ to $200 \mathrm{~m}$ 


\subsection{Sediment Layer Effect}

The model was also applied to quantify the effects of various sediment configurations. The sediment configurations of interest are the position of the dense, highly reflective Troutdale formation and the composition of the top layer. The effect of the Troutdale formation depth was studied by computing solutions with variable bedrock depths that were otherwise equivalent. Figures 5.6 and 5.7 show the results of the bedrock layer comparisons. Variations in the shallow water bedrock has the greatest effect, while bedrock layers of depths greater than $25 \mathrm{~m}$ have little practical effect on the SELs present in the water column for the medium sand top layer of the Columbia River. The $15.5 \mathrm{~m}$ bedrock depth curve shown corresponds to the layer depth used in the comparisons to acoustic data.

The effect of the top layer is analyzed by comparing simulated results using various published parameters, describing alternate sediment compositions. The parameters used for comparison of the top sediment layer are summarized in Table 5.1. Sediments with large sand portions were emphasized to examine the effects of mixing the sandy bottom of the Columbia River with additional soil components. All parameters are standard values from the literature [27].

The result of the top layer comparison is shown in Figures 5.8 and 5.9. In general, the curves show a complex relationship between density and attenuation on SEL, with neither sound speed nor density having a dominant effect. The Sediment 


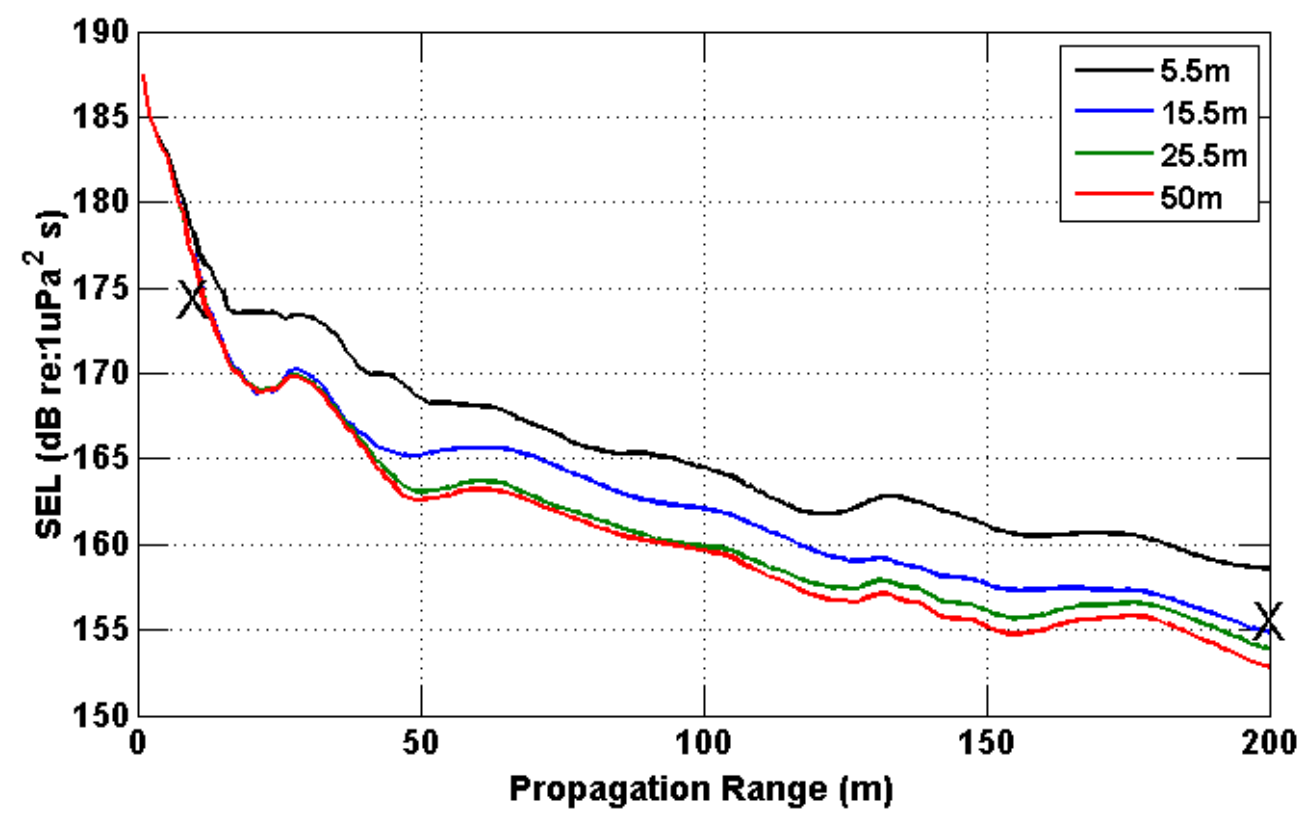

Figure 5.6: Range dependant SEL at 3.5m depth for multiple bedrock depths about pile B1. X demarcates the acoustic observations. Bedrock depths are relative to the simulation bathymetry.

Table 5.1: Parameters for Top Sediment Layer Comparison

\begin{tabular}{|l|l|l|l|}
\cline { 2 - 4 } \multicolumn{1}{c|}{} & Attenuation $d B / \lambda$ & Density $\mathrm{g} / \mathrm{cm}^{3}$ & Sound Speed $\mathrm{m} / \mathrm{s}$ \\
\hline Sandy Clay & 0.0890 & 1.147 & 1420 \\
\hline Sandy Mud & 0.2107 & 1.49 & 1420 \\
\hline Sandy Gravel & 0.9306 & 2.492 & 1936 \\
\hline Coarse Silt & 1.177 & 1.195 & 1472 \\
\hline
\end{tabular}

layer curves are similarly shaped for each sediment configuration except sandy clay, where there is a dramatic spike in SEL at about $370 \mathrm{~m}$. In the case of sandy clay, the signal is attenuated very little in the sediment, and the large spike peaking at $370 \mathrm{~m}$ range corresponds to the reflection of the first arrival off from the Troutdale formation, which is at a depth of $51.5 \mathrm{~m}$ for pile A3. This is diagramed in Figure 5.10 , where the reflection of the first arrival from the bedrock layer can be seen to 


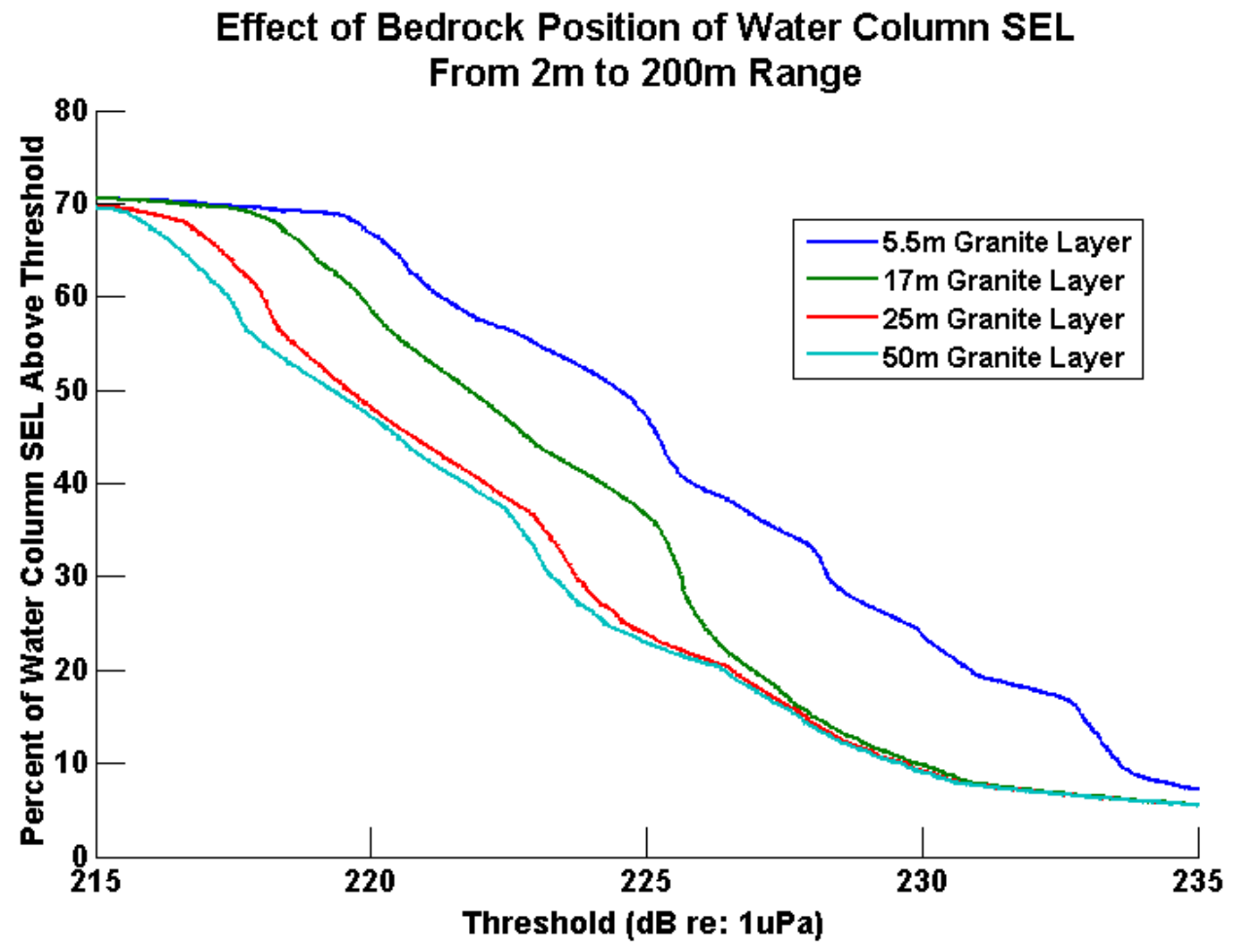

Figure 5.7: Statistical threshold plot comparing the percentage of the water column above certain SELs, for several bedrock depths. Again, variations in a shallow bedrock layer have the greatest effect, and bedrocks below $25 \mathrm{~m}$ depth have little practical effect on the water column SEL. The region of consideration is the water column from range $100 \mathrm{~m}$ to $200 \mathrm{~m}$.

reach the receiver at approximately $371 \mathrm{~m}$. This is an important result, because it predicts that dramatic SEL spikes are possible at long range for environments that include low attenuation and density top layers, coupled with dense bedrock layers, even if those bedrock layers are very deep. 


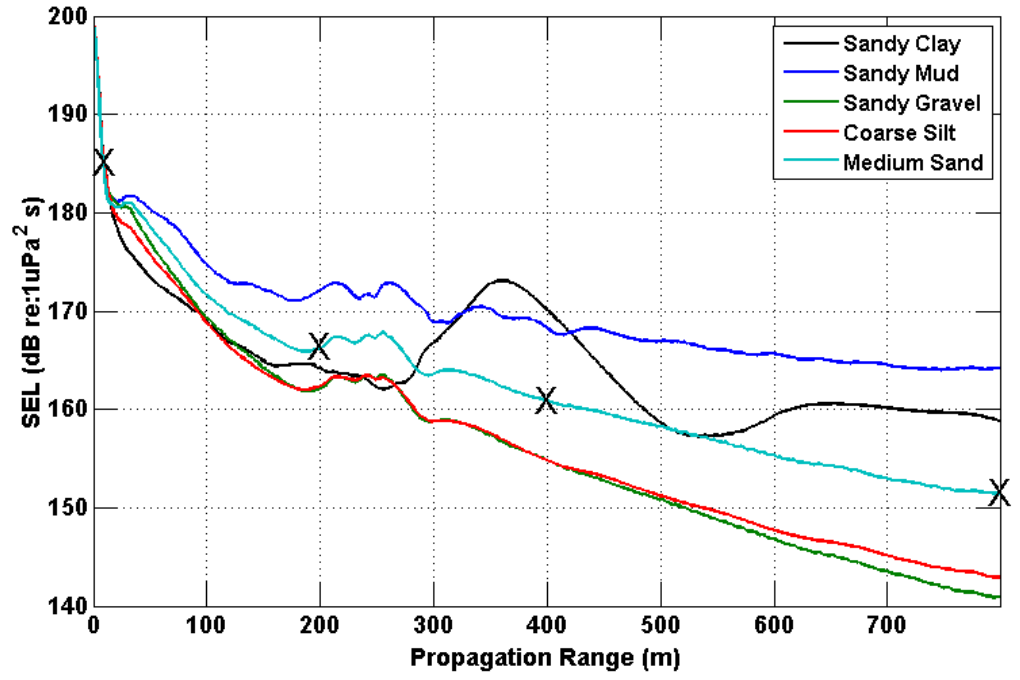

Figure 5.8: Range dependant SEL at 3.5m depth, for the top layer compositions in Table 5.1, about pile A3. X marks the observed SEL.

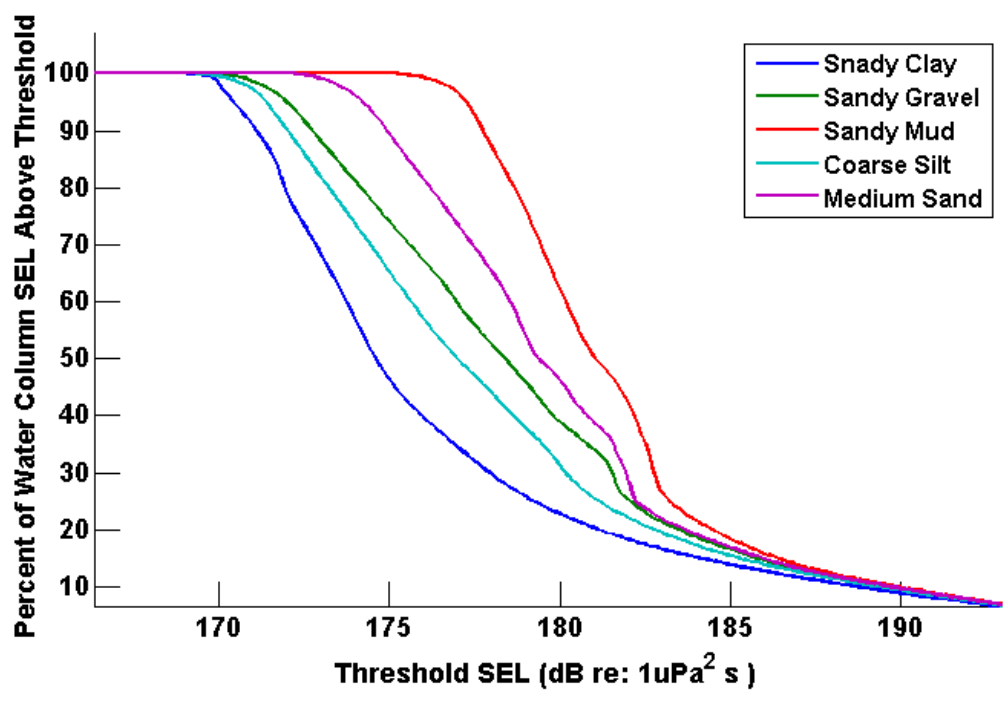

Figure 5.9: Statistical threshold plot comparing the percentage of the water column about certain SEL, for each top layer composition. The area of interest is the water column from range $100 \mathrm{~m}$ to $200 \mathrm{~m}$. 


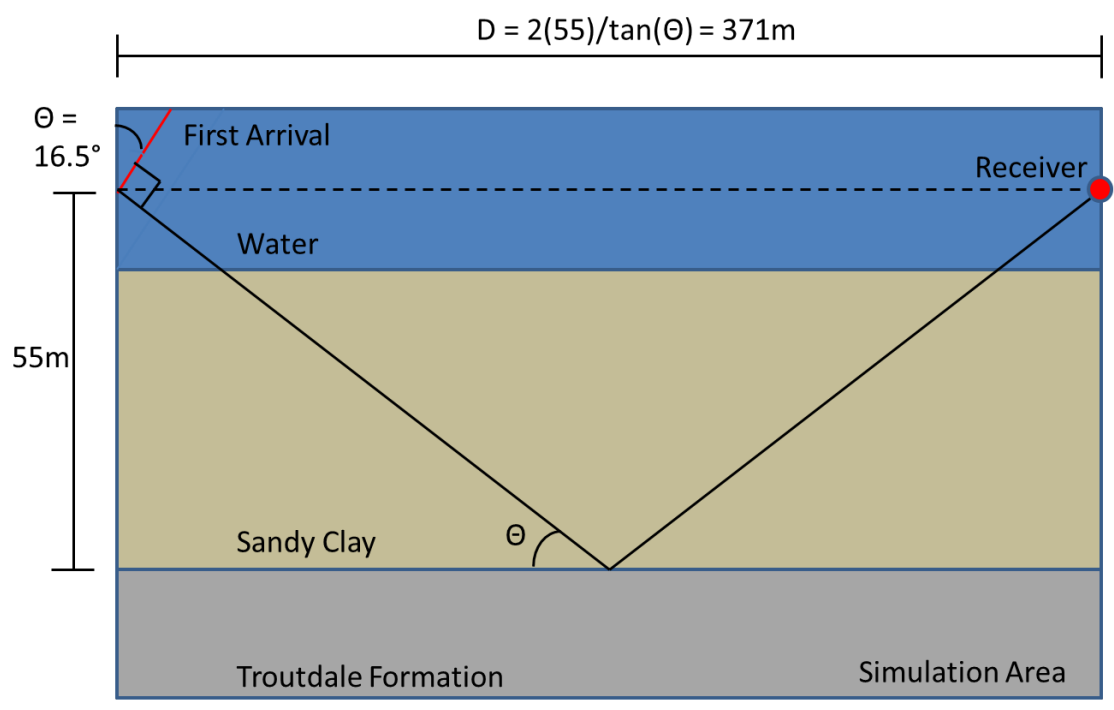

Figure 5.10: Geometrical explanation for the large SEL spike seen around $370 \mathrm{~m}$ in Figure 5.8. The propagation angle is assumed to change very little in the sediment due to the similar sound speeds. 


\section{Chapter 6}

\section{Conclusion and Future Work}

\subsection{Conculsion}

Underwater impact pile driving is the source of increasing environmental and regulatory concern due the extremely high underwater sound levels radiated into the surrounding environment, which are known to harm marine wildlife. In this thesis the noise from underwater impact pile driving was analyzed with a computational model that used physical models of the pile driving source, coupled to a broadband synthesis of the RAM-PE, a PE based wave propagation tool. Source models included an empirical model from Reinhall and Dahl [2] and an FDTD model from

Shahab and Hastings [5]. These source models were coupled with convergent results produced by the RAM-PE that used a sediment model and bathymetry that are range-dependent.

The computational model was applied to the Columbia River environment between Portland, OR and Vancouver, WA, which was advantageous due to the environmental monitoring and site characterization done in preparation for the construction of the new I5 span. Environmental monitoring included acoustic observations of test pile observations in the North and South riversides performed by the consulting firm David Evans and Associates. Acoustic waveforms due to pile 
driving of 24 and 48 inch piles were recorded at 5 locations each, at ranges from 10 to $800 \mathrm{~m}$, which provided close and long range comparisons between the model and observation, providing 20 comparisons.

The model showed strong agreement with observations in PSD, SPL, and SEL. PSD comparisons show levels and roll off that are typically in very good agreement. Most of the features in the observed data are captured in SPL comparisons at close range, and at longer propagation ranges, the levels are accurately predicted. SEL agrees within $4 \mathrm{~dB}$ at all but 3 of 25 comparisons, and usually the agreement is much better, to within $2 \mathrm{~dB}$. Within $400 \mathrm{~m}$ range, SEL shows very good agreement at all observation locations.

Beyond comparisons to acoustic data, the model was applied to produce SEL contour plots over large areas of the Columbia River environment and study the effects of bathymetry and sediment configurations. The absolute depth of the bathymetry is found to be the only factor that significantly affects long-range sound levels, while other variations create only localized effects. Also, the bedrock layer is determined to be insignificant when deeper than $25 \mathrm{~m}$ below the mud line for the medium sand layer present in this region of the Columbia River.

\subsection{Future Work}

While the model showed strong agreement in the Columbia River environment near the new Columbia River Crossing, it has not been tested in other environments. 
Future work should apply the model to environments with bathymetry and sediment composition differing from the environment presented in this thesis. Furthermore the present modeling does not take into account the attenuation effects of shear waves, and should be included. Also, the FDTD analysis used was limited and not of a completed nature, and future work should expand and evaluate the effectiveness of the FDTD source model. Finally, further optimization is possible, especially with the FDTD source model, and further work should address this. Particularly great optimization is possible by producing convergent $\mathrm{PE}$ simulations that calculate the contributions of several nodes in a single PE execution. 


\section{References}

[1] M.C. Hastings and A.N. Popper, "Effects of Sound on Fish," California Department of Transportation Contract No. 43A0139, (2005).

[2] P.G. Reinhall and P.H Dahl, "Underwater Mach wave radiation from impact pile driving: Theory and observation," J. Acoust. Soc. Am. 130(3) (2011).

[3] M.D. Collins, "A split-step Padé solution for the parabolic equation method," J. Acoust. Soc. Am. 93, 1736-1742, (1993).

[4] C. Erbe, "Underwater Noise From Pile Driving in Moreton Bay, QLD," Acoustics Australia 37(3), 87-92 (2009).

[5] S. Shahab and M.C. Hastings, "Transient analysis of sound radiated from a partially submerged cylindrical pile under impact," J. Acoust. Soc. Am. 132(3), 2034, (2011).

[6] S. Hardyniec and S. Skeen, "Pile Driving and Barotrauma Effects," Journal of the Transportation Research Board, 184-190 (2005).

[7] C. Mueller-Blenkle, P.K. McGregor, A.B. Gill, M.H. Andersson, J. Metcalfe, V. Bendall, P. Sigray, D. Wood, and F. Thomsen, "Effects of pile-driving noise 
on the behaviour of marine fish," COWRIE Ref: Fish 06-08 / Cefas Ref: C3371 Technical (2010).

[8] J.A. David, "Likely sensitivity of bottlenose dolphins to pile-driving noise," Wanter and Enviornment Journal, 20, 48-54, (2006).

[9] J. Carstensen, O.D. Henriksen, and J. Teilmann, "Impacts of offshore wind farm construction on harbour porpoises: acoustic monitoring of echolocation activity using porpoise detectors (T-PODs)," Mar. Ecol. Prog. Ser. 321, 295308 (2006).

[10] J.A. Reyff, "Underwater Sound Pressure Levels Associated with Marine Pile Driving," Journal of the Transportation Research Board,CD 11-S, Transportation Research Board of the National Academies, Washington, D.C., pp. 481490, (2005).

[11] S.P. Robinson, P.A. Lepper, and J. Ablitt,, "The measurement of the underwater radiated noise from marine piling including characterisation of a soft start period," IEEE Oceans, 978-1-4244-0635-7 (2007).

[12] M.L. Stockham, P.G. Reinhall, and P.H. Dahl, "Characterizing Underwater Noise from Industrial Pile Driving at Close Range," IEEE, 978-1-4244-4332-1 (2010). 
[13] B. Würsig, C.R. Greene, and T.A. Jefferson, "Development of an air bubble curtain to reduce underwater noise of percussive piling," Marine Environmental Research, 49, 79-93 (2000).

[14] A. Stokes, K. Cockrell, J. Wilson, D. Davis, and D. Warwick, "Mitigation of Underwater Pile Driving Noise During Offshore Construction: Final Report," Department of the Interior, Minerals Management Service, Contract Number: M09PC00019 (2010).

[15] ICF Jones and Stokes, "Technical Guidance for Assessment and Mitigation of the Hydroacoustic Effects of Pile Driving on Fish," California Department of Transportation, (2009).

[16] F.B. Jensen, W. A. Kuperman M.B. Porter, and H. Schnidt, Computational Ocean Acoustics, (AIP, New York, 1994)

[17] E.C. Ifeachor and B.W. Jervis, "Digital Signal Processing: A Practical Approach," Addison-Wesley, New York, (1993).

[18] M.C. Junger, "Acoustic fluid-elastic structure interactions: basic concepts, computational structures." 65(3), 287-293 (1997).

[19] A.J. Edmondson, "Solution of shell problems by the finite difference method," Nuclear Engineering Design 11(2), 208-216 (1970). 
[20] J.E. Goldberg and T. Korman, "A finite difference formulation of general shell equations," Nuclear Engineering Design 26(3), 440-443 (1974).

[21] G.B. Warburton, "Vibration of a cylindrical shell in an acoustic medium," Proc. Inst. of Mech. Eng. Part C-J. Eng. Mech. Eng. Sci. 3, 69-79 (1961).

[22] L.E. Kinsler, A.R. Frey, A.B. Coppens, and J.V. Sanders, Fundamentals of Acoustics, (Wiley, New York, 2000)

[23] Columbia River Crossing, "CRC Geotech Reports: Appendix A Boring Logs,"

[24] Columbia River Crossing, "CRC Geotech Reports: Appendix F Laboratory,"

[25] David Evans and Associates, "Columbia River Crossing Test Pile Project Hydroacoustic Monitoring Final Report," Columbia River Crossing agreement number Y-9246, Task AH, Amendment No. 7 (2011).

[26] E.L. Hamilton, "Geoacoustic modeling of the sea floor," J. Acoust. Soc. Am. 68(5), 1313-1340 (1980).

[27] APL-UW High-Frequency Ocean Environmental Acoustic Models Handbook, Applied Physics Laboratory, University of Washington (1994).

[28] http:www.ram.nrl.navy.mil/ftp/pub/ram 


\section{Appendix A}

\section{Computational Model Algorithm}

This appendix discusses the details of the computational model implementation, and should be a sufficient reference for further modeling work. The computational pile driving model uses a primary propagation code, and depending on the source model, pre-processing and post-processing routines. For both source models, the result is a 3 dimensional matrix of depth, range and frequency, which defines the broadband propagation over a 2 dimensional slice of environment. Single or multiple $2 \mathrm{D}$ solutions were processed to produce graphical and quantitative results, in the time and frequency domain, for comparisons with data and prediction of sound levels. All modeling was implemented in the MATLAB language.

\section{A.1 FDTD Source Pre-Processing}

The FDTD pre-processing routine calculated the initial pressure solutions, defined by equation (2.31), for convolution with the RAM-PE Green's functions. First, the time domain particle velocity solution was read in from the Excel spread sheet, provided by GTech. For each node, the time domain solution was zero padded to accommodate the user specified frequency resolution (to correspond to the required broadband frequency resolution), transformed to the frequency domain via the DFT, and multiplied by the depth dependent specific acoustic impedance (2.29) 
as well as the other scaling factors in the expression for the starting fields (2.31). The pressure spectrum at each node is truncated to the user entered bandwidth requirements and windowed using a slight Tukey window (window factor of 0.015) to reduce ringing in the time domain synthesis. Finally, the result is saved along with the frequency and depth axis into a file to be called by the propagation code.

\section{A.2 Broadband Propagation Model}

Broadband propagation was handled using the publicly available code titled the RAM-PE. This code can be found at [28]. The RAM-PE is a FORTRAN code, and when executed draws the model parameters from an input script, which varied slightly depending on the source model. The parameters defined by the script were grouped into simulation, transect and environment parameters. Simulation parameters included the solution destination folder, the solution file name, and the range and depth span to be saved in the final solution. For the empirical source model, the number of arrivals modeled, depths of the point source solutions and bandwidth parameters were also defined. The FDTD source required that the source data file name be specified as well as which source depths were calculated. Since the contributions from source depths combine linearly, modeling only certain sources was useful for breaking up simulations over multiple computers. Transect parameters defined the source location, simulation bearing angle, and simulation length. 
The environment parameters specified the geoacoustic model. The sediment depths $(\mathrm{m})$, densities $\left(\frac{g}{c c}\right)$, sound speeds $\left(\frac{m}{s}\right)$ and attenuation $\left(\frac{d B}{\lambda}\right)$ were specified in matrices, where each row defined the parameter for the sediment layer corresponding to the layer at the depth defined in the sediment spacing matrix. The model allowed an arbitrary number of range dependent updates in the geoacoustic model, which corresponded to the columns in the environmental parameter matrices. The ranges of these updates were specified in the update range matrix, where parameters were linearly interpolated between updates. Finally, the wetted pile length, total pile length and pile sound speed were specified for the empirical model.

The model first extracted the bathymetry using the source location, bearing angle and length from the Columbia River bathymetry data. The bathymetry simulation and environment data were then passed to the main propagation routine, which calculated the broadband propagation. This code executed RAM-PE for each of the frequencies, source depths and arrivals defined in the input file. For the empirical source model, it was only necessary to calculate the first two arrivals. Since RAM-PE is a FORTRAN code, it was compiled as an executable (which required certain cygwin dlls to be present) that was called using a system command in the MATLAB code. The input to RAM-PE is handled by the use of a text file, which was written for each RAM-PE execution by the MATLAB code. RAM-PE also outputs a text file, which is read in by the model and arranged into a matrix of depth of range. Since RAM-PE does not include the phase change associated 
with the movement of the wave front, a range dependent phase shift was applied to the solution. Finally, the result was truncated in range and depth to the user specified bounds, and placed in the three dimensional output matrix. This matrix was saved with the depth, range and frequency axis, as well as the bathymetry and sediment spacing information. The empirical source model saved each arrival in separate files.

\section{A.3 Empirical Source Post-Processing}

For the empirical source, the propagation code alone calculated the phased Greens functions, summed over all source depths, for the first two arrivals, without offsets or spectral weighting. That is, the first two arrivals, produced by the ringing bulge, of flat spectrum and equal, unit amplitude. The empirical post processing routine used these pieces to assemble to final simulation. The user entered parameters included the file names of the arrivals, the attenuation factor (3.2), the spectral weighting function and the offset parameter (2.27), the number of arrivals to include, and the name of the completed simulation. Since the odd and even arrivals differ only in an attenuation constant and time delay, only the first two arrivals needed to be calculated to compute a complete simulation, with an arbitrary number of arrivals.

The code first read in the observed waveform and calculated the spectral weighting function. The full simulation was assembled by looping through all arrivals, applying the attenuation constant and spectral weighting function, applying any 
additional extra time delay (depending on the arrival), then summing the result into the final solution matrix. Finally the offset was calculated using the observed waveform (2.27). The offset was applied to the complete simulation and the results saved. At this point, from a numerical standpoint, the FDTD and empirical source model results were identical. 


\section{Appendix B}

\section{RAM-PE Convergence}

The accuracy of solutions produced using the PE method is dependant on the convergence of the solution. That is, the $q$ term rational approximation in equation (2.10) converges to the correct solution of the forward differential equation (2.8), for the given frequency and environmental configuration (i.e. bathymetry and sediment layers). Convergence is dependent upon the choice of certain input parameters. The parameters of most importance are the range and depth grid spacing, $d r$ and $d z$, but the number of stability constraints and number of Padé terms (the number of terms in the $q$ term rational approximation (2.8)) can also affect convergence. Typically, general rules are followed to obtain a correct grid spacing, such as $d z=\frac{\lambda}{10}$, but due to the requirement of broadband analysis, it was advantageous to obtain a uniform grid for all frequencies, and therefore avoid errors due to interpolation.

In general, finer spatial resolutions lead to convergence, however, especially for the low frequency, longer wavelength simulations; erroneous results can results from an over-fine spatial grid. Furthermore, a given grid can cause single frequency solutions to lose convergence entirely and produce extremely erroneous solutions. The cause of these glitches was the failure of the operator in (2.9) to satisfy the equation in forward differential equation (2.8), which was usually alleviated by changing the number of Padé terms calculated. Due to this, convergent parameters 
needed to be determined not only at the minimum and maximum frequencies, but also at several frequencies in the bandwidth. Furthermore, it was necessary to check each frequency solution for convergence. With these considerations, a uniform grid was defined for all frequencies, but also a frequency dependent number of Padé terms was also used (i.e. 500-1500 Hz, 5 Pad terms). Two stability constraints were used in all modeling.

The method for obtaining convergent parameters is called the convergence test. In this method, identical simulations are calculated, varying only the range and depth resolutions. The solutions that match those calculated with different spatial grids are convergent. Range and depth steps are varied by powers of 2 across a specified range, and for each range step, a solution is calculated for each depth step.

The convergence test method is illustrated in Figure B.1. Comparing Panels (a) and (b), there is very little difference between the output results, despite the different spatial grid sized. In the bottom of Panel (b), the TL curve from Panel (a) has been overlaid with the curve taken from Panel (b), and no difference can be seen. In Panel (c) however, the new curve is dramatically different, and this spatial grid is not considered to be convergent. This process is then repeated using different numbers of Padé terms and stability constraints in order to obtain a uniform convergent grid over all frequencies. 
(a)
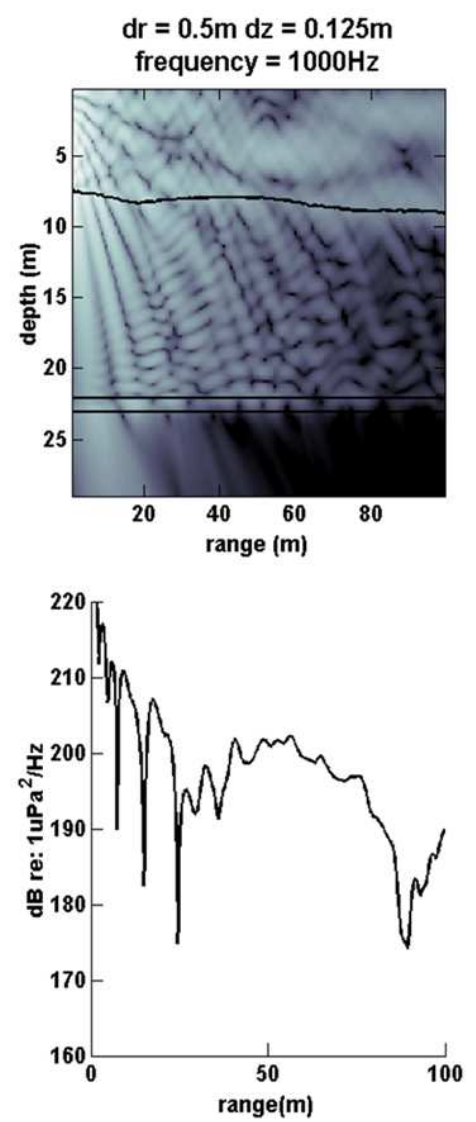

(b)
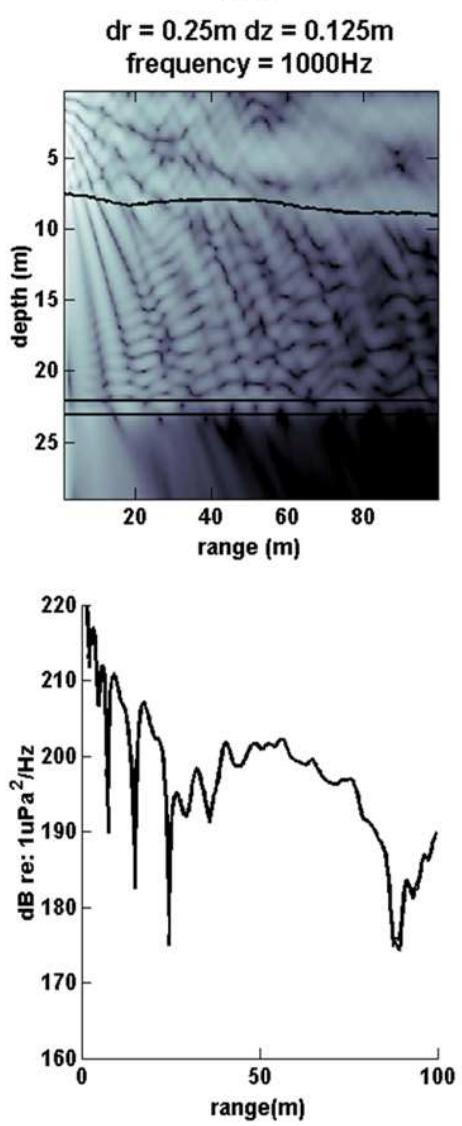

(c)
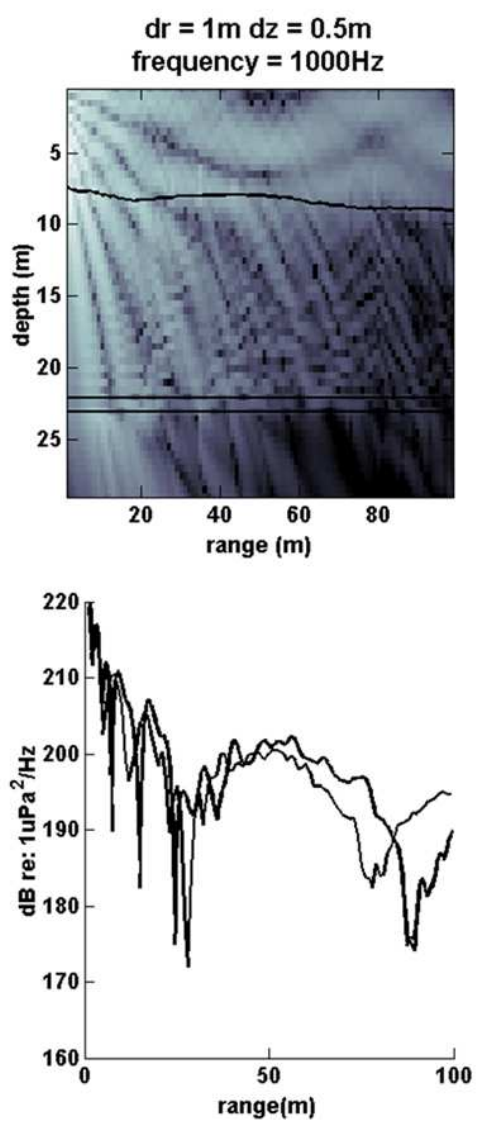

Figure B.1: Illustration of the convergence testing method. Panel (a), (b), and (c) show the results of otherwise identical simulations computed using different spatial grids, where the grid sized increase from left to right. The curves at the bottom of each panel are the TL lifted from the upper part of the frame at a depth of $4.5 \mathrm{~m}$. 


\section{Appendix C}

\section{Model Optimization}

For the empirical source model, the self-starter (2.12) was calculated repeatedly for sev

Due to the broadband, multi-source nature of the computational model, the model is very computationally demanding. Typically, a single RAM-PE execution for a propagation range of $800 \mathrm{~m}$ requires $45 \mathrm{~s}$ of $\mathrm{CPU}$ time. Computing such propagation for the typically 1700 discrete frequencies and 100 source depths leads to extremely long computation times, on the order of a week. While, due to the linearity inherent in the model, simulations can be easily broken up and calculated on several computers in parallel, the computation times are still large and pose a significant obstacle. This was alleviated somewhat by optimization of the model. The two main increases in efficiency came by use of the MATLAB parallel computing toolbox as well as modifications of the RAM-PE Fortran code.

\section{C.1 Parallel Computing}

The MATLAB parallel computing toolbox was developed to better utilize multiple processor cores, which are becoming increasingly prevalent. To do this, it allows certain operations to be broken up and computed on several cores independently, through the use of what is called a parfor (parallel for) loop. In the propagation 
code, the parfor loop is used on the frequency loop, such that all frequency components of the broadband simulation are computed in parallel. The implementation of the parfor loop required that all frequency dependent parameters be calculated prior to the use of the parfor loop, in arrays dependent on a single index. Also, since RAM input and output is done through unique text files, a system for creating, deleting and accessing unique directories for the text files was developed. In all of the code, the parallel computing toolbox is available to use by default. To use the parallel computing toolbox, the user must only first set up the multiple core work group using the matlabpool command. The use of a 4 core processor group archived an approximately $3 x$ increase in efficiency.

\section{C.2 RAM-PE Source Code Modification}

The greatest efficiency improvement resulted from modification of the RAM-PE FORTRAN code in order to reduce the number of executions. The goal of these modifications was to circumvent the need to execute RAM to compute the contributions from each individual sources, but rather compute all source depths in a single execution. The implementation of this was different depending on the source model. eral source depths rather than the default single source depth. Furthermore the depth-dependent phase shifts (2.23) were calculated by RAM, and applied to the corresponding source depths. Theses solutions were summed together and used to produce the starting fields. The parameters to be used for these calculations 
were passed to RAM by modification of the input script to specify the source depth positions (first source depth, number of source depths and source depth spacing), pile characteristics (pile wetted and total length; pile sound speed), and arrival number. The efficiency improvement from this method was directly proportional to the number of source depths, typically a speed up of 100x.

For the FDTD model, the self-starter was circumvented entirely, and the fields generated by (2.31) were used directly in place of the self-starter. This was implemented by the use of a second input text file, which contained the starting fields. While this approach showed promise for significant efficiency gains, convergent results were not obtained with the RAM-PE for these modifications. Due to this, this approach was not used. 